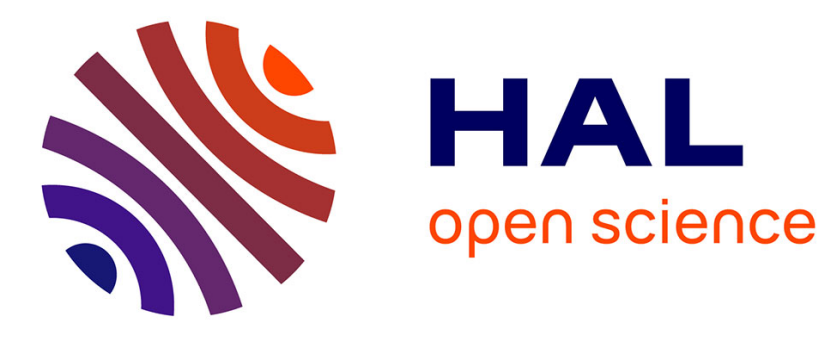

\title{
Testing With Bars From Dynamic to Quasi-static Gérard Gary
}

\section{To cite this version:}

Gérard Gary. Testing With Bars From Dynamic to Quasi-static. CISM Udine Italy. Constitutive Relations of Materials under Impact Loadings: Experiments, Theoretical and Numerical Aspects., CISM Udine Italy, pp.1-59, 2013, Summer School. hal-00968913

\section{HAL Id: hal-00968913 https://hal.science/hal-00968913}

Submitted on 1 Apr 2014

HAL is a multi-disciplinary open access archive for the deposit and dissemination of scientific research documents, whether they are published or not. The documents may come from teaching and research institutions in France or abroad, or from public or private research centers.
L'archive ouverte pluridisciplinaire HAL, est destinée au dépôt et à la diffusion de documents scientifiques de niveau recherche, publiés ou non, émanant des établissements d'enseignement et de recherche français ou étrangers, des laboratoires publics ou privés. 


\title{
Testing With Bars From Dynamic to Quasi-static
}

\author{
Grard Gary \\ Laboratoire de Mcanique des Solides, Ecole Polytechnique, Palaiseau, France
}

\begin{abstract}
The numerical calculation of the dynamic loading of a structure includes a great number of steps in which various fundamental or engineering problems are involved. Most of them are addressed in the present course at CISM. In this paper, we discuss the testing of materials in order to model their behaviour.

Because of waves induced in the testing device by impulse loadings and short time measurements, the data analysis has to deal with transient effects. Using bars makes easier such an analysis. For this reason, Hopkinson bars are a very commonly used dynamic testing device.

Using the word "dynamic" means that "time" is considered as an active parameter in the evolution process. When dynamically testing a structure (a cylindrical specimen is a common example of such a structure) the effects of time appears in different ways.

There is not static equilibrium in the machine so that measurements at specimen ends cannot be simply deduced from measurements with sensors incorporated in the machine, as it is the case with quasi-static testing. Furthermore, most sensors (like force cells) have a limited high passing band.

Transient effects in the specimen induce waves and the nonhomogeneity of mechanical parameters. Consequently, average or global measurements cannot be right away related to local ones.

Stresses cannot be simply related to forces measurements as inertia effects are also involved - the most known effect is the confinement induced by lateral inertia, especially important when testing a big specimen of brittle material.

Short testing times do no allow for isothermal testing - a metallic specimen can have a temperature increase up to $100^{\circ} \mathrm{C}$ during a SHBP test.

The behaviour of an elementary volume of the material can depend on the rate of change of basic mechanical parameters strain and/or stress. This last effect (strain rate sensitivity) is the (only) one that is expected to be measured, in most cases.
\end{abstract}


In (dynamic) mechanical testing it is then suitable to consider separately the global measurements made on the specimen (forces applied at a part of the specimen border and displacement measured at another - or the same - part) and the analysis of its mechanical evolution.

This is commonly done in the quasi-static side but is not always, for historical reasons, done in dynamic testing.

The above discussion does not answer the basic question of the boarder between quasi-static and dynamic testing. Theoretically, indeed, waves in solids are still present in quasi-static testing. The common criterion to evaluate this limit is to compare the time $\tau_{e}$ needed to reach equilibrium (say $<5 \%$ of non homogeneity of stresses and strains) to the measurement duration. Note that $\tau_{e}$ mostly depends on the specimen size and on the elastic speed of waves in the material and not on the measurement duration. In the classical SHPB literature, this problem is related to the "impedance matching problem", misunderstood in many publications, perfectly addressed in 1963 by Davies \& Hunter. Based on this criterion, (too) many SHPB tests are considered as quasi-static ones.

\section{Why using bars}

Taking account of the transient response of the machine seems a difficult theoretical problem as far as a geometrically complex loading machine is considered. Two ways allow for avoiding this difficulty.

The first one is to make measurements directly at specimen faces. Even if modern optical devices can provide direct displacement measurements, force measurements need transducers in which mechanical waves are induced by the loading.

The second is to use a simple enough machine allowing for an analysis of transient effects. This is the case of Hopkinson bars.

In order to illustrate the problems encountered with a classical machine, we examine the case of a drop test.

We consider the simplified description of a drop test machine using, as an example, the compression test of an aluminium honey comb. As a first approximation, we assume that the force response of the specimen provides a constant value $\mathrm{F}_{0}$ (fig. 1).

In this simulation, the force is measured in two different ways. We first consider a linear spring the shortening of which is measured by an instantaneous optical device. Secondly, the force is deduced from the deceleration of the known falling mass. The corresponding acceleration is measured with an accelerometer of finite size in its middle. For sake of simplicity, the test 
is modeled with the one-dimensional analysis. In this case, usual relations between jumps of stress, particle velocity and strain are used. See basic demonstration later or, in a more general frame, Achenbach (1978).

$$
\Delta \nu=-c \Delta \varepsilon \quad \Delta \sigma=-\rho c \Delta v
$$

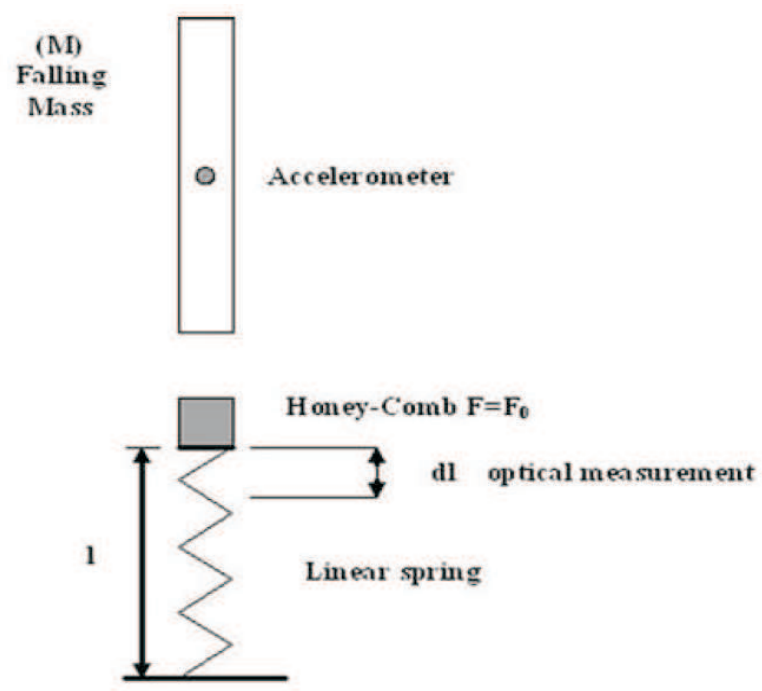

Fig. 1. One-dimensional scheme of a drop test.

At any time, the stress and the particle velocity can be calculated at any section of the falling mass and of the spring. Fig. 2 shows the calculated velocities at the accelerometer position and at the head of the spring.

Knowing the velocity of the accelerometer, its acceleration is obtained by derivation. To avoid obtaining an infinite acceleration, the speed considered is the average speed across the accelerometer and it depends on its size. If the falling mass is supposed to have this measured deceleration, a measured force is deduced.

From the velocity of the springhead, the displacement is obtained by integration and the relative variation of the length of the spring is known. If the force supported by the spring is supposed proportional to this displacement, another measured force is deduced.

Both forces, as they would be deduced from a quasi-static analysis of the test, are shown in fig. 3 . 


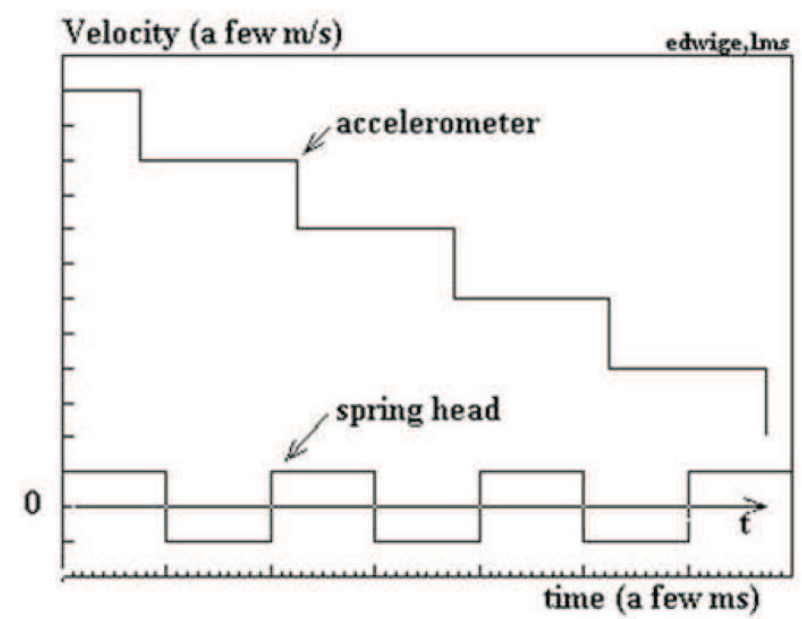

Fig. 2. Velocity at measuring points.

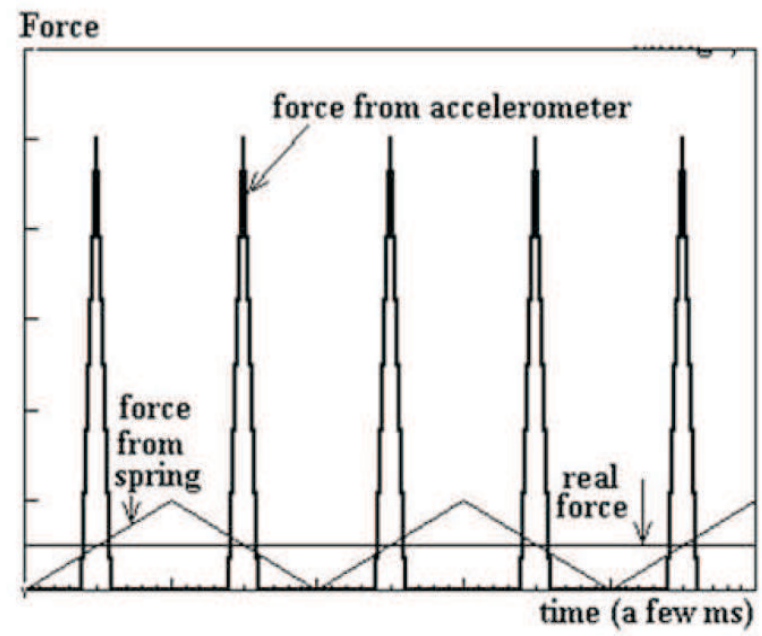

Fig. 3. "measured forces"

It is observed that the two methods give very different results. In both cases, the maximum force is over estimated, especially with the acceleration measurement. Nevertheless, it can be checked that the mean value of both force measurements corresponds to the exact one. It confirms that a quasistatic analysis of the test could be done provided that the duration of the 
test is great compared with the oscillation period of each system and that the passing band of the system is adapted.

This example purposely goes to extremes in order to show the importance of transient effects in dynamic testing. Such an analysis largely explains the difference observed between the two results of crash-tests done on the same structure (square tube) with an industrial drop test machine and, in our laboratory with a Hopkinson bar. Both results are shown together in fig. 4, with same scales on axes.
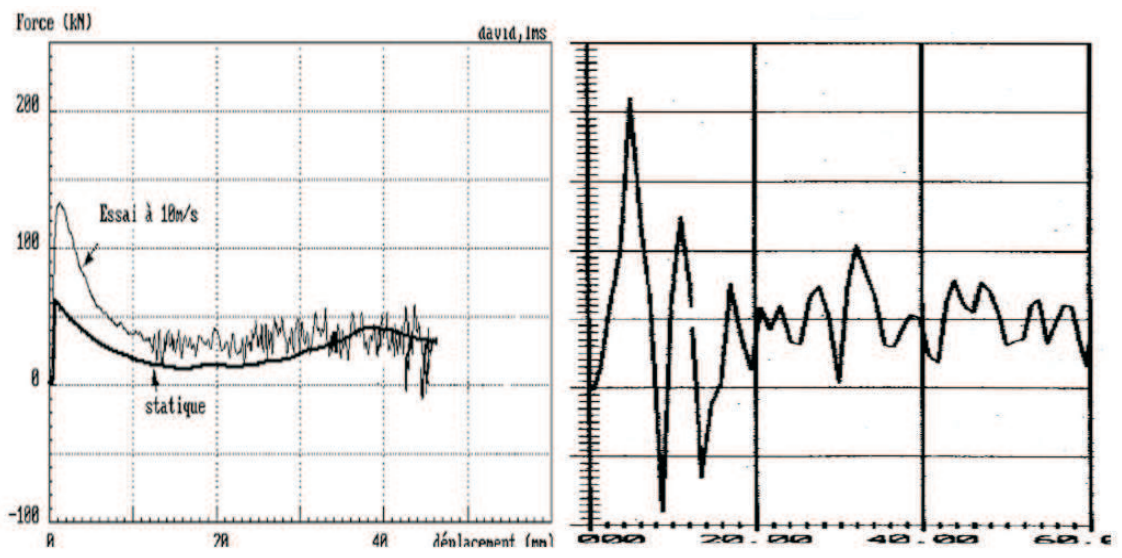

Fig. 4. Measurement with Hopkinson bars (left), falling mass (right)

Looking at fig. 4, one observes that, as expected, the dynamic response of the square tube shows a greater force than the static response of the same tube. The dynamic result also shows a good agreement with a numerical simulation of the test. The test duration of the Hopkinson bar system $(6 \mathrm{~m}$ long aluminium input bar, diameter $80 \mathrm{~mm}$ ) has been increased by using a deconvolution technique that will be described later.

This example shows how important are transient and inertia effects in dynamic testing machines and it also shows that a good account of them is taken with Hopkinson bars. There are various other dynamic testing techniques that will not be considered in this paper as we focus on the Hopkinson bars, or Kolsky apparatus. 


\section{Basic notions on 1-D waves}

\subsection{Dalembert's equation}

The general form of the so called "Dalembert's equation" concerns one time variable $t$ and one or more spatial variables $x_{1}, x_{2}, \ldots, x_{n}$, and a function $u=u\left(x_{1}, x_{2}, \ldots x_{n}, t\right)$, the values of which model some amplitude of a wave.

The wave equation for $u$ is $\frac{\partial^{2} u}{\partial t^{2}}=c^{2} \nabla u$, where $\nabla$ is the (spatial) Laplacian and where $c$ is a fixed constant.

In solid mechanics, and within the Lagrangian formalism where variables are related to material points, the generic scalar function is the amplitude of the displacement vector at a point.

In the particular case of 1-D elastic bars, this displacement is a scalar where $c$ is the speed of the 1-D wave. The wave equation can be then established in a very simple manner.

Let us consider the dynamic equilibrium a thin slice of a bar with a thickness $d x$ between abscissa $x$ and $x+d x$.

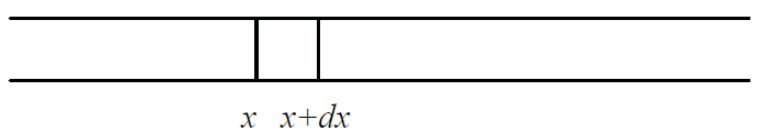

$A$ is the area of the bar, $\rho$ its density, $\sigma$ the uniaxial stress, $u$ the displacement, $\varepsilon$ the uniaxial strain.

Dynamic equilibrium $(F=m \gamma)$ for this slice reads:

$$
A \sigma(x+d x)-A \sigma(x)=\rho A d x \frac{\partial^{2} u}{\partial t^{2}}
$$

Using elasticity 1-D $(\sigma=E \varepsilon)$ and the definition of 1 -D $\operatorname{strain}(\varepsilon=$ $\partial u / \partial x)$ one obtains:

$$
A E \frac{\partial \varepsilon}{\partial x} d x=\rho A d x \frac{\partial^{2} u}{\partial t^{2}}
$$

and the Dalembert's 1-D equation:

$$
\frac{\partial^{2} u}{\partial t^{2}}=c^{2} \frac{\partial^{2} u}{\partial x^{2}} \text { with } c^{2}=\frac{E}{\rho}
$$

Both terms support to be differentiated with respect to $x$, so that Dalembert's equation is also verified by the uniaxial strain (and by the linearly 
linked uniaxial stress). In mechanical applications the equation of waves more often considers the strain.

The general solution of this equation is known to be in the form:

$$
u=f(x-c t)+g(x+c t)
$$

where $f$ and $g$ are arbitrary functions.

The variable $x-c t$ means that the value of $u$ at point $x$ and time $t$ is the same as at an other position $x^{\prime}$ and an other time $t^{\prime}$ such as

$x-c t=x^{\prime}-c t^{\prime}$ or $x-x^{\prime}=c\left(t-t^{\prime}\right)$

The value $u$ is observed at a distance $\left(x-x^{\prime}\right)$ after a delay $\left(x-x^{\prime}\right) / c$. It defines the propagation of a signal in one direction at speed $c$.

The variable $x+c t$ defines, in a similar way, the propagation in the opposite direction.

\subsection{Relations between strain, stress and particle velocity}

These relations can be defined as a particular case of "jump equations" established for material waves.

An easier and more comprehensive way to derive them is to recall the general form of a single wave propagating in the positive direction (conventionally defined) and its derivatives with respect to space and time.

$$
u=f(x-c t) \quad \varepsilon=\frac{\partial u}{\partial x}=f^{\prime}(x-c t) \quad v=\frac{\partial u}{\partial t}=-c f^{\prime}(x-c t)
$$

For a wave propagating in the positive direction, on has then.

$v=-c \varepsilon$ and (from $\left.E=\rho c^{2}\right) \sigma=-\rho c v$

On has to keep in mind that these relations are valid in a Galilean referential. Considering for instance the shock of a striker on a bar needs to carefully account for the initial speed of the striker. In terms of jumps, equations (2) are recovered.

$$
\Delta \nu=-c \Delta \varepsilon \quad \Delta \sigma=-\rho c \Delta v
$$

Equations (2), together with the propagation relations, are the foundations of Hopkinson bar measurements, where forces and displacements are computed at bar end on the base of strain measurements.

\section{Real bars: dispersion relations}

\subsection{Case of elastic bars}

\section{A philosophical point}


From an epistemological point of view, it is interesting to observe that, in the general 3-D elastic case, one can find two solutions only of the Dalembert's equation corresponding to the so called $\mathbf{P}$ and $\mathbf{S}$ waves. By reference to seismology, $\mathbf{P}$ is for "premires" (first arrived, speed $c_{p}$ ) and $\mathbf{S}$ for "secondes" (speed $c_{s}$ ).

$$
c_{p}=\sqrt{\frac{E(1-\nu)}{\rho(1+\nu)(1-2 \nu)}} \quad c_{s}=\sqrt{\frac{E}{2 \rho(1+\nu))}}
$$

For a Young's modulus equal to $200 \mathrm{GPa}$, a Poisson's ratio 0.3 and a density $7850 \mathrm{~kg} / \mathrm{m} 3$ (values for steel), $c_{p}=5860 \mathrm{~m} / \mathrm{s}, c_{s}=3130 \mathrm{~m} / \mathrm{s}$, while the speed of the 1-D wave is $5047 \mathrm{~m} / \mathrm{s}$.

Note that for torsion bars, the speed of the torsion wave is $c_{s}$.

The 1-D wave (that theoretically does not exist, as no bar is purely 1D) has a speed in between 3-D speeds and is a theoretical concept that describes very well the wave signals observed in bars. Such a "wave" could be also considered as a mixture of 3-D waves traveling at speeds $c_{p}$ and $c_{s}$.

\section{Dispersion}

Knowing that the 1-D model is not perfect, as a careful observation of waves might show it at once, it is worth looking at a more complete model.

The main point is that, due to a positive value of Poisson's ratio, the compression goes with a diameter increase inducing lateral inertia effects.

On the contrary, shear waves (torsion waves in torsion bars), which do not induce volume change, are non dispersive.

The wave dispersion effects (due to radial expansion) on the longitudinal elastic wave propagating in cylindrical bars have been studied experimentally by Davies (1948). On the basis of the longitudinal wave solution for an infinite cylindrical elastic bar given by Pochhammer's (1876) and Chree (1889), a dispersion correction has been proposed and verified by the experimental data. Even though the Pochhammer-Chree solution is not the exact one for a finite bar, it is found easily applicable and sufficiently accurate see Davies (1948).

In the Pochhammer-Chree's longitudinal wave analysis, it is assumed that the wave is harmonic as follows.

$$
\mathrm{u}(r, z, t)=\frac{1}{2 \pi} \int_{-\infty}^{+\infty} \overline{\mathrm{u}}(r, \omega) e^{i[\xi(\omega) z-\omega t]} d \omega
$$

where $\mathrm{u}(r, z, t)$ is the displacement vector.

The complete solution of the governing equations with the boundary condition on the external surface of the bars leads to a frequency equation that gives a relation between the wave number $\xi$ and the pulsation $\omega$. 


$$
\begin{aligned}
f(\xi)= & \left(2 \alpha / r_{0}\right)\left(\beta^{2}+\xi^{2}\right) J_{1}\left(\alpha . r_{0}\right) J_{1}\left(\beta . r_{0}\right)-\left(\beta^{2}-\xi^{2}\right)^{2} J_{0}\left(\alpha . r_{0}\right) J_{1}\left(\beta . r_{0}\right) \\
& -4 \xi^{2} \alpha . \beta . J_{1}\left(\alpha . r_{0}\right) J_{0}\left(\beta . r_{0}\right)=0
\end{aligned}
$$

with $\alpha^{2}=\frac{\rho \omega^{2}}{\lambda+2 \mu}-\xi^{2} ; \beta^{2}=\frac{\rho \omega^{2}}{\mu}-\xi^{2} ;$ and $J_{0}, J_{1}$ are the Bessel functions, $\lambda$ and $\mu$ are the elastic coefficients, $r_{0}$ is the radius of the bar.

It is important to note that this equation has an infinite set of solutions which are called the propagating modes. As they do not define a basis of a vector space, (they are not orthogonal to each other) it cannot be decided, from a single measurement, how the energy is split into the different modes.

A very nice point is that, at low frequencies, only one mode has a real celerity. The frequency at which could appear the second mode is around $0.22 c / a$ (for steel, with a radius equal to $10 \mathrm{~mm}, f_{\text {cut }}=105 \mathrm{kHz}$ ). It can be checked that such a frequency is generally above the spectrum of a standard test (even without pulse shaper).

It is then of common use with Hopkinson bars to consider the first mode solution of equation (4). This use has been carefully validated by Tyas \& Watson (2001).

This harmonic wave solution has been studied numerically by Bancroft (1941). Bancroft's data is given in the form where the phase velocity variation $C / C_{0}$ is a function of Poisson's coefficient $\nu$ and of the ratio between radius and wave length $r_{0} / \lambda$ for the non dimensional interest.

$$
C / C_{0}=F\left(r_{0} / \lambda, v\right),
$$

with $C=\omega / \xi$ and $\lambda=2 \pi / \xi$

The previous works - Follansbee \& Franz (1983), Gorham (1983), Gong et al.(1990), Lifshitz \& Leber (1994)- use this data to correct the wave dispersion in bars. Following Yew \& Chen's works (1978), they calculate harmonic components in frequency domain of signals by Fast Fourier Transform (FFT) and find the phase difference for each component from (Eqn. 4). The corrected signals in time domain can be recovered from the corrected frequency components.

Their correction procedure in term of variation of phase velocity can be re-written as follows. From the knowledge of the dispersion relation between wave number $\xi$ and frequency $\omega$ given by the solution of (4) or (5), one can calculate, from a measured wave $u_{z}^{m}(t)$, the wave $u_{z}^{i}(t)$ reached at a distance $\Delta z$. Using the components in $\mathrm{z}$ of the formula (2) at the surface of the bar, one can write $u_{z}^{m}(t)$ and $u_{z}^{i}(t)$.

$$
\mathrm{u}_{z}^{m}(t)=\frac{1}{2 \pi} \int_{-\infty}^{+\infty} \overline{\mathrm{u}}_{z}\left(r_{0}, \omega\right) e^{i\left[\xi(\omega) z_{0}-\omega t\right]} d \omega
$$




$$
\mathrm{u}_{z}^{i}(t)=\frac{1}{2 \pi} \int_{-\infty}^{+\infty} \overline{\mathrm{u}}_{z}\left(r_{0}, \omega\right) e^{i\left[\xi(\omega)\left(z_{0} \Delta z\right)-\omega t\right]} d \omega
$$

The wave shifting procedure can be then performed numerically by the FFT.

$$
u_{z}^{i}(t)=F F T^{-1}\left[e^{i \xi(\omega) \Delta z} F F T\left[u_{z}^{m}(t)\right]\right]
$$

Accordingly, the correction accuracy depends only on that of the dispersion relation $\xi(\omega)$. The use of Bancroft's data must be done carefully as one has to take care of the following points. First, as the value is given only in form of a table for a certain Poisson's coefficient and for certain values of the ratio between radius and wave length $r_{0} / \lambda$, which is known discretely, an interpolation is needed. Second, equation (5) only gives an implicit relation for correction, between wave number $\xi$ and frequency $\omega$. It is then recommended to calculate directly from (4) the dispersion relation, in the form of wave's number $\xi$ as function of $\omega$. for a given Poisson's ratio and Young's modulus.

The application of formula (7) with modern computers is instantaneous and should be systematically used. Using "pulse-shapers" to reduce the high frequencies in the recorded signals (and consequently reducing the need of the correction) also reduces the rising time of the signals and then the duration of the commonly found "constant plateau".

It is observed that the dispersion changes the slope of the signal in the early stage of the test and has a sensitive effect on the average stress-strain curve in the range of small strains, as shown by Gary et al. (1991) and Zhao and placeCityGary (1996).

A good way to test the quality of the dispersion relation is to check whether the oscillations induced by the dispersion disappear at the impact side where the signal can be transported, as shown in fig. 5 .

The two "ears" are due to two plastic rings used to guide the striker. (The non-nul slope is not explained)

Another way is to calculate the stress at a free end, which must be zero, as shown in fig. 6 . 


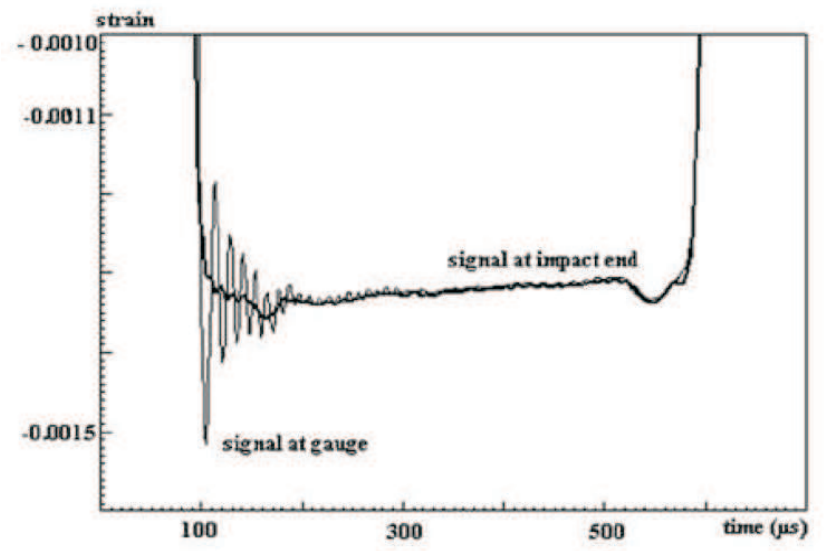

Fig. 5. Incident signal transported at striker side. (striker $1.3 \mathrm{~m}$ and input bar $3 \mathrm{~m}$ made of steel, diameter $20 \mathrm{~mm}$. Incident gauge in the middle, striker speed $13.3 \mathrm{~m} / \mathrm{s}$ )

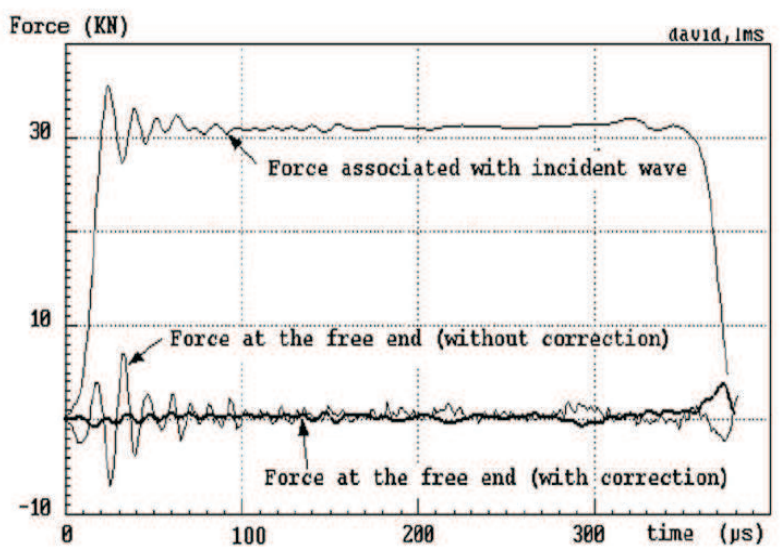

Fig. 6. The forces at a free end.

\subsection{Case of visco-elastic bars}

For viscoelastic bars, the argument $\xi$ in equation (4) is a complex number so that a solution cannot be expressed in a simplified form such as (5). Consequently, the computation of the dispersion relation needs the functions $\lambda^{*}(\omega)$ and $\mu^{*}(\omega)$ that describe the viscoelastic behaviour of the bars.

The material properties of standard viscoelastic materials are often represented with a rheological model. The simplest one (called Zenner model) 
needs 3 parameters to be identified (first 3 left elements in figure 7 ).

They could be identified in different ways, more often when dealing with bars by an inverse approach based on the measurement of the wave propagation on the bar itself. This is a safe method as, unlike for metals, material properties depend on various external factors.

Zhao and Gary (1995) have used an inverse method based on such an idea. They found that the Zenner model was not complete enough, and had to use a more complex model with 9 parameters (figure 7). Furthermore, following many authors, they assumed a constant Poisson's ratio.

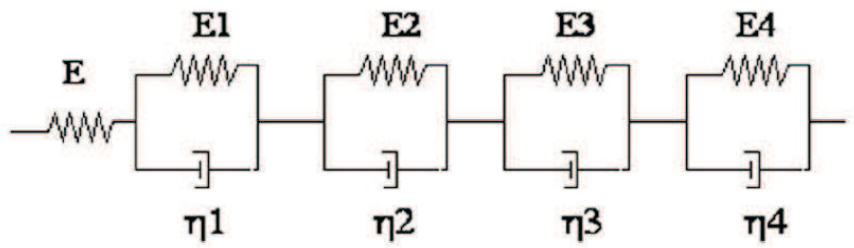

Fig. 7. A linear viscoelastic model

The same experimental set-up as in an impulse test is applied, where the waves are recorded at two different points in the bar. Using the wave at a recording point as input data, the parameters are determined in comparing the predicted wave and the recorded wave at another point (like in fig. 8)

It is also possible to avoid to solve equation (4). Dispersion may indeed be experimentally determined by comparing the wave Fourier components measured on two points on the rod - Blanc (1971), Lundberg and Blanc (1988), Gorham (1983), Bacon (1998). More recently, Hillstrm \& al. (2000) developed a multi-point method using least squares. Another method, very accurate, is also proposed by Othman \& al. (2001) based on a one-point measurement method using a spectral analysis of the resonant frequencies of the rod. An recent extension and improvement of this method proposed by Collet et al (2012) is presented in chapter 7 .

In order to illustrate the excellent quality of the dispersion relations generally obtained, an original record and two other records at respective distances of $4 \mathrm{~m}$ and $8 \mathrm{~m}$ of the original one, compared with their predictions, are shown in fig. 8.

By comparison with the elastic case (see fig. 4) it is observed that the dispersion correction is more important for common size viscoelastic bars and could not be neglected.

The importance of the correction in a real test situation is illustrated by showing both forces calculated in a test without specimen where the output force must obviously be equal to the input one (fig. 9 and 10). 


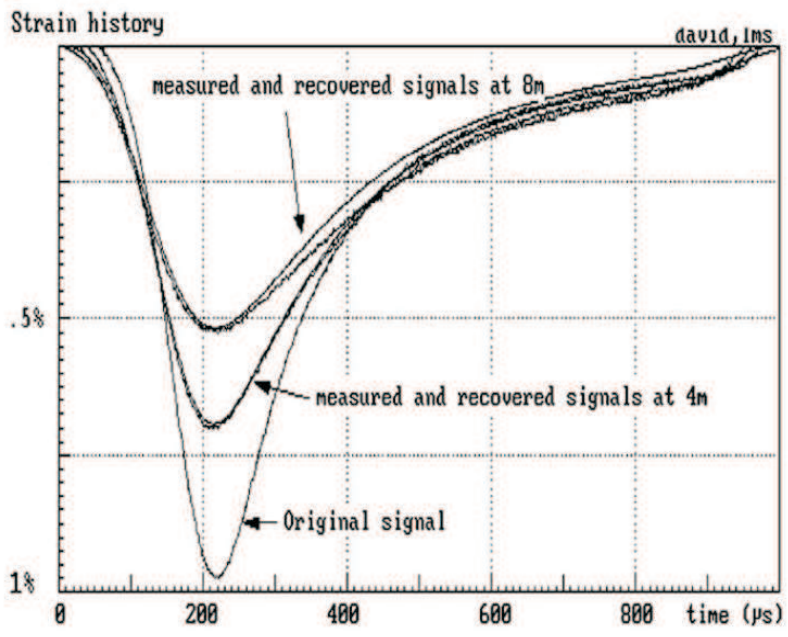

Fig. 8. Test of the dispersion relation.

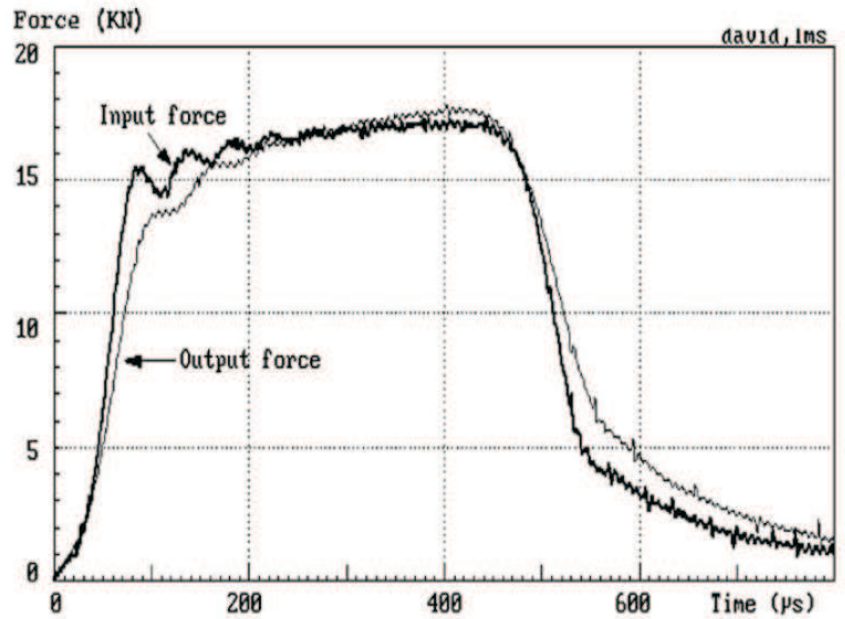

Fig. 9. Input and output forces without dipersion correction.

For both viscoelastic and elastic bars, a precise dispersion correction does not suppress oscillations of the incident force in the case of sharp loading pulses. These oscillations are indeed the physical consequence of the wave dispersion.

At very high speeds of loading with short specimens, oscillations can also be seen in the output signal 


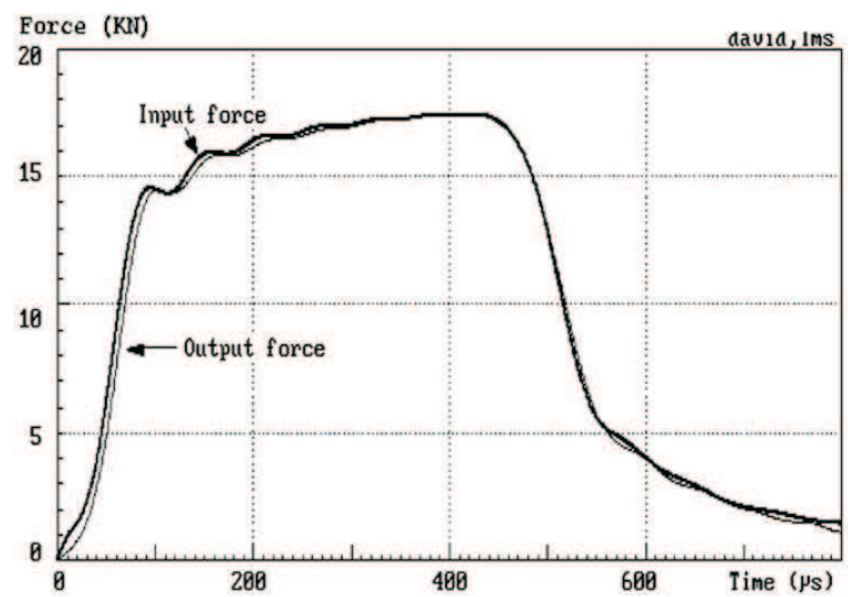

Fig. 10. Input and output forces with dipersion correction.

\subsection{Planerity of waves}

The planarity of waves is extensively studied by Davies for the first mode. The solution of equation (4) is well known and illustrated in his paper for particular values of the frequency, as shown in figure 11 - from Davies (1948).
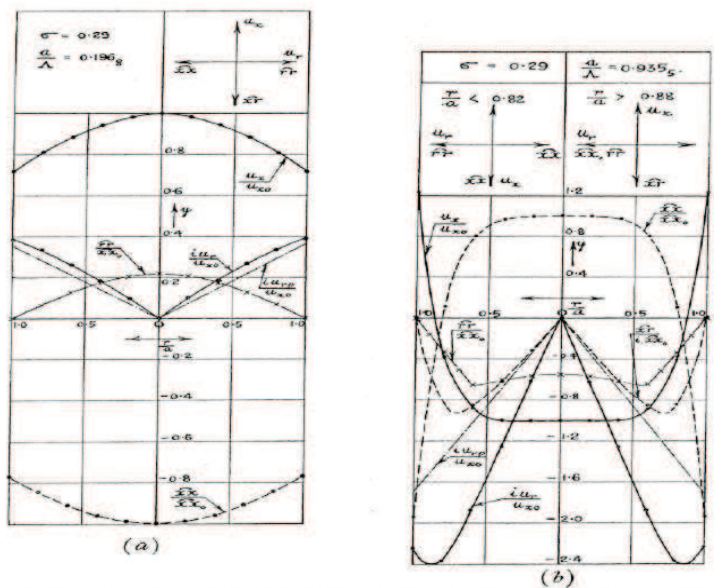

Ftoure 19. The variation of the displacements and the stresses over the cross-section of a bar of radius $a, \widehat{x}=$ longitudinal stress; $\widehat{x r}=$ shearing stress; $\widehat{r}=$ radial stress; $u_{x}=$ longitudinal displace

Fig. 11. Stresses and strains function of radius and frequency. 
In this figure one sees, among other variables, the relative variation of the stress along the radius $\left(x x / x x_{0}\right)$. It shows that, at a relative high frequency (in 19b, 0.935 corresponds to a non realistic value of the frequency for our standard steel bar: $280 \mathrm{kHz}$ ), the stress at the surface of the bar is negative when the average stress is positive. At a more realistic frequency (figure $19 \mathrm{a}-94 \mathrm{kHz}$ for our bar) one can see that the average stress is more or less $10 \%$ higher than the stress at the surface. The strain is not proportional to the strain, but those observations are also valid for the average strain. On has then to keep in mind that, depending on the frequency:

a) - The strain measured at the surface is not proportional to the average strain.

b) - The axial strain and the orthoradial strain are not proportional to each other.

c) - The axial stress is not proportional to the axial strain.

d) - The average axial speed is not proportional to the axial strain.

Point (b) is well illustrated by Tyas and Watson (2001).

At lower frequencies, all the measurements become close to be proportional to each other.

A few authors - Safford (1992), Merle and Zhao (2006) have checked the validity of usual hypothesis commonly used. They proved that it is not worth taking care when frequencies $f$ are under $2 c / a$ (where $a$ is the radius of the bar, equivalent for steel, to $a / \lambda<0.2$ ). For the standard $20 \mathrm{~mm}$ steel bar, one observes that frequencies are in the good range.

\section{Direct impact test}

Recall here that, with non strictly $1 \_D$ bars.

The average strain is not proportional to the strain measured at the surface of the bar. The average stress is not proportional to the average strain

The dispersion of the waves induces significant changes in rising times of the signals.

The displacement at bar end is over estimated as the punching of the bar by the specimen is neglected (this point will be addressed later).

In the following, the basic understanding of SHB is recalled based on the 1-D propagation theory.

A convenient way to have a good understanding of waves propagation and reflections in a system of bars is to use the "Lagrange" representation together with formulas (2). In a space-time (abscissa-ordinate) diagram, the fact that the celerity of waves is constant induces that a wave event follows a straight line with an absolute slope $c$ (figure 12). 


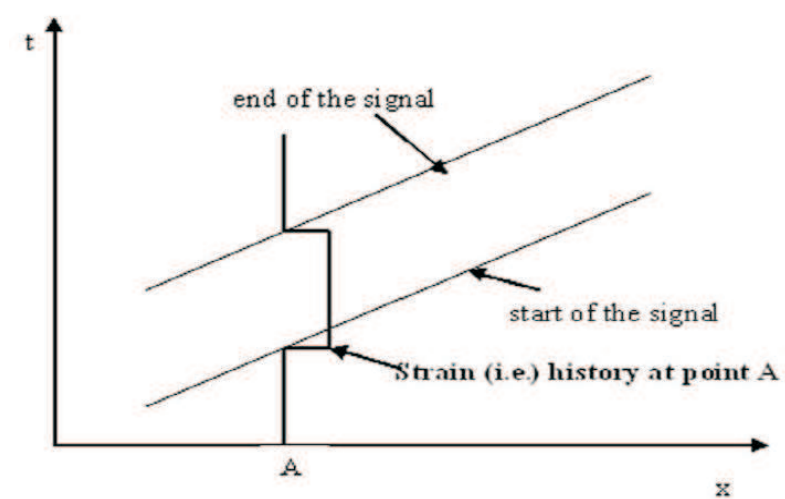

Fig. 12. A typical Lagrange diagram. The two sloping lines show the path of the beginning and the end of a square signal in the space-time diagram. At a given point A, the time story show what could have been recorded by a gauge at this point.

\section{Direct impact test: 1 force and 1 displacement measurements.}

A force is applied at the end of a bar of length $l$ starting at time $t=0$. It produces a strain-time signal which is measured by a gauge at a (short) distance $a$ of the bar end. The corresponding signal $\varepsilon_{i}(t)$ starts at time $t_{s}=a / c$. The beginning of the signal reaches the other end (say output) of the bar at a time $t_{e}=l / c$.

A given boundary condition is applied at the output end of the bar. In order to satisfy this (unknown) boundary condition, a new wave $\varepsilon_{r}(t)$ in the opposite direction is produced which starts at time $t_{e}$.

This reflected wave reaches point A at time $t_{r}=l / c+(l-a) / c$.

From this time $t_{r}$, the strain measured by the gauge is the sum of the initial wave and of the unknown reflected one.

Consequently, the measurement duration based on the record of the initial wave is $\Delta t_{m}=t_{r}-t_{s}=2(l-a) / c$.

Say the strain measured at the gauge is $\varepsilon_{m}(t)$.

The measurement duration is limited by the overlapping of waves.

During this time $\Delta t_{m}$,

The strain at bar (input) end is $\varepsilon(t)=\varepsilon_{m}(t-a / c)$

The force at input bar is $F=E_{b} S_{b} \varepsilon$

The speed at input bar is $v=-c \varepsilon$ 


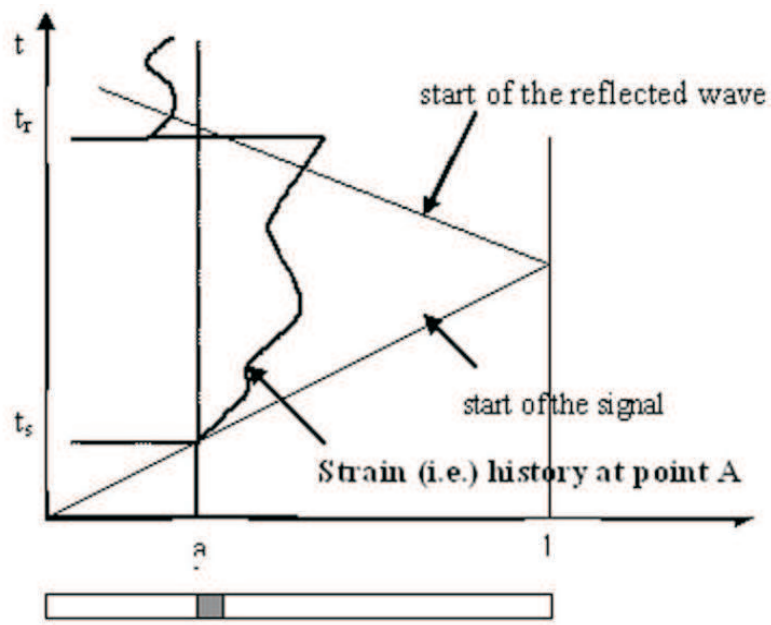

Fig. 13. A Lagrange diagram for an impact test.

If

- one side of the specimen is fixed at the input end,

- the speed of the other side of the specimen can be measured,

- the equilibrium of the specimen is assumed,

\section{Then,}

The force-(relative) displacement relation of the specimen can be computed.

\section{$5 \mathrm{SH}(\mathrm{P}) \mathrm{B} .2$ forces and 2 displacements measurements}

The specimen is sandwiched between two bars. One force and one speed measurement are made on each bar-end in contact with the specimen. The output bars works as for the direct impact. It is not the case for the input bar where, due to the loading, there exists a wave prior to the loading of the specimen itself.

Torsion bars and direct tension bars will not be explicitly addressed here. Their analysis is based on the same knowledge of basic wave propagation theory, but they need to solve some specific technical problems concerning the loading of the system and the specimen clamping.

In this chapter, some problems related to the measurement side (that of forces and speeds) will be addressed. Anyway, due to the very common use of Kolsky's formulas, this historical case is briefly recalled first, where problems dealing with the specimen behaviour have to be also introduced. 


\subsection{The special case of Kolsky bars}

This very special case is interesting. For historical reasons, the gauges are equally distant from the specimen and both bars are identical. Considering the values of the strain at specimen ends, forces and displacements at both specimen ends are given by formulas $(7,8)$

$$
\begin{array}{cc}
v_{i}=-c_{b}\left(\varepsilon_{i}-\varepsilon_{r}\right) & v_{o}=-c_{b}\left(\varepsilon_{t}\right) \\
F_{i}=A_{b} E_{b}\left(\varepsilon_{i}+\varepsilon_{r}\right) & F_{o}=A_{b} E_{b} \varepsilon_{t}
\end{array}
$$

Where $v_{i}, v_{o}, F_{i}, F_{o}$ are input and output speeds and input and output forces at specimen faces, respectively. $A b, E b, c b$ are area, Young's modulus and celerity of waves in (identical) bars, respectively.

$\varepsilon_{i}, \varepsilon_{r}, \varepsilon_{t}$ are incident, reflected and transmitted waves, respectively, computed at specimen faces.

The measurement finishes here. In particular, the "Kolsky" formulas that will be discussed later are resulting of more assumptions, mainly: identical bars and specimen equilibrium. This second assumption is always an approximation.

The situation is indeed here exactly the same as any measurement where the machine basically provides forces and displacements. Consequently it is possible, without any lost in the quality of measurements, to use different bars.

In Kolsky's standard processing, the special assumption of equilibrium is used in the case of identical (same material, same diameter) bars. This yields - along with the superposition principle and formulas $(7,8)$ - the well known relation:

$$
\varepsilon_{i}(t)+\varepsilon_{r}(t)=\varepsilon_{t}(t)
$$

Consequently, assuming the homogeneity of stresses and strains within the specimen, the incident wave does not explicitly appear in the simplified formulas:

$$
\varepsilon_{s}(t)=\int_{0}^{t} \dot{\varepsilon}_{s}(\tau) d \tau=-\frac{2 c_{b}}{l_{s}} \int_{0} \varepsilon_{r}(\tau) d \tau \quad \sigma_{s}(t)=\frac{A_{b}}{A_{s}} E_{b} \varepsilon_{t}(t)
$$

where $A_{s}$ is the area of the specimen.

These simplified formulas are both based on equilibrium. When equilibrium is only badly verified, some researchers suggest the use an average formula for the stress (sometimes called the "three waves formula"). First, one must keep in mind that the average force could give a worth response than one of other input force or output force. Second, the simplified formula for the strain could also produce an imperfect result. 


\subsection{Overlapping of waves}

The best way to address easily this problem is to make use of Lagrange diagram. It already has been seen for the direct impact test which corresponds, with the Kolsky apparatus, to the output bar. When equilibrium is not assumed and has to be checked, a measure of both input and output forces is needed. In this case, the best position for the input gauge appears to be in the middle of the input bar. Consequently the length of the output bar is such that the length between the gauge and its end is half of the input bar. If the bars do not have the same impedance, one must take care of propagation time and not of length.

Theoretically, the length of the striker could be as long as half of the input bar. In real situations one must account that the duration of the input wave is greater than that of the theoretical "square" one. The rising time of the signal has indeed to be added. For a given striker, this time is related to the imperfect geometrical matching of both striker and input bar ends. This mismatch can be due to imperfect surfacing and/or imperfect alignment but it can be considered equivalent to an imperfection of a constant thickness. A realistic interpretation of the rising time is that of the time needed for the bars to flatten the geometric mismatch.

In the case of a standard steel SHPB $20 \mathrm{~mm}$, the rising time is around $20 \mu \mathrm{s}$ with a striker speed of $10 \mathrm{~m} / \mathrm{s}$. It should be around $100 \mu \mathrm{s}$ at a striker speed of $2 \mathrm{~m} / \mathrm{s}$.

If the minimum loading speed is $2 \mathrm{~m} / \mathrm{s}$, these consideration lead to the standard optimal SHPB configuration, with:

Input bar length $=2 l$

Output bar length $=l+5 d$ (5 diameters form the end for the gauge position)

Striker length $=l-0.25 \mathrm{~m}$ (theoretical loading duration decreased by $100 \mathrm{~s})$

Note that for "pulse shaper" users, the rising time will increase and depend on the "pulse shaper" geometry and material.

In the case of viscoelastic bars and a viscoelastic striker, the analysis is a little different. With a striker and an input bar having the same impedance, the loading duration (in the 1-D analysis) is infinite, due to a tail. It is not easy to decide when the amplitude of the tail is small enough. It is then recommended, with viscoelastic strikers and bars, to use a striker with a smaller (by around 10\%) diameter than the bars. Bussac et al. (2008) have indeed demonstrated that the incident pulse has, in this case, a finite duration. 


\subsection{Wave shifting. A precise method for SHPB.}

The 1-D analysis of the waves implicitly takes account of the SaintVenant principle: a certain distance is needed between the end of the bar and the strain gauge to insure the homogeneity of the strain across the bar (typically 5 diameters).

One needs then to use a wave theory to deduce the strain (as it would be is this point was not an end) at the end a bar. This operation is called shifting.

It is clear that the input force is proportional to the sum of the incident and reflected waves and that a relative imperfect shifting in time would induce an error, especially at the beginning of the loading. Some authors have proposed to use some geometrical trick at the beginning of the signals to define a "foot" for the curves and determine the shifting process on the knowledge of these points: we call that the "foot shifting" technique. Acceptable results could be obtained for the input force, with this method.

If we extend the method to the output wave, it induces a wrong evaluation, at least, of the strain, if it neglects the times needed by the loading to go across the specimen.

The shifting process must then be precise. A method, introduced by Zhao \& Gary (1996), can be used. It is based on the transient simulation of an initial elastic behaviour of the specimen. The method also allows for a correction of specimen geometry and a measurement of the Young's modulus of the specimen material.

The incident wave at the input specimen face been known (after the shifting process), reflected and transmitted waves can be computed - depending on specimen dimensions, bar dimensions and mechanical properties, specimen Young's modulus. The only unknown is the last one. Using a try and error method, one rapidly finds the Young's modulus that gives shapes of both simulated waves similar to that of transmitted and reflected waves as they are known (after the shifting process) at the input and output specimen faces.

Note that this operation does not work if the dispersion is not taken into account, even with elastic bars. The reason is that the elastic response of the specimen concerns the first instants of the loading where the initial slope is strongly affected by the dispersion.

Some distance in time is still often observed between real and simulated waves. This distance can be due to an imperfect knowledge of the speed of waves or, more often, to some geometrical imperfection of the specimen. For an optimized stress-strain curve, real waves are shifted to simulated ones. This very short shift (equivalent of a few $\mathrm{cm}$ in distance) is not affected by the dispersion. An illustration of the method is presented in figures 14 and 
15.

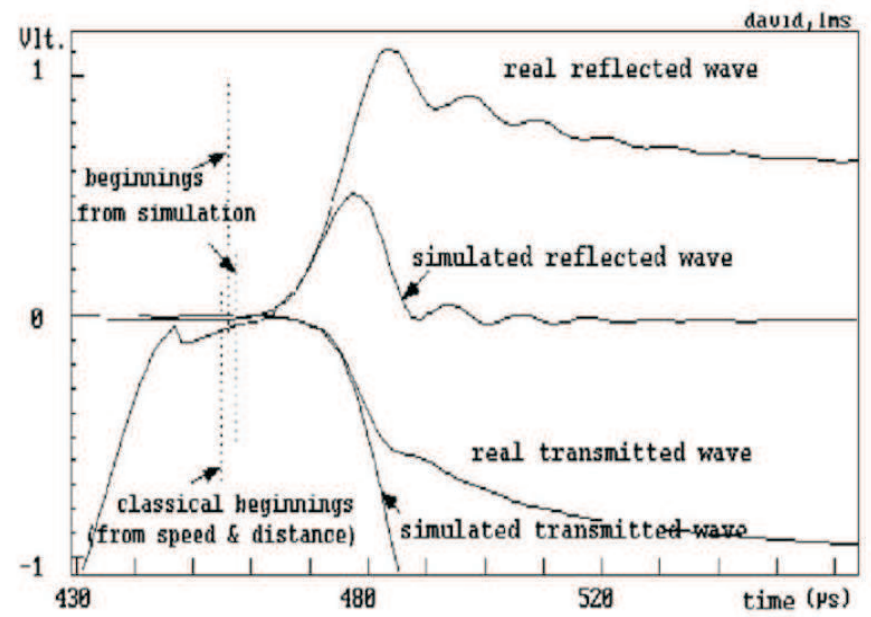

Fig. 14. Optimal choice of Young's modulus for transmitted and reflected waves.

The precise way stress and strain are computed will be addressed later. Remind that the best results are derived from the force and displacement time histories at the specimen boundaries without artificial time shifts.

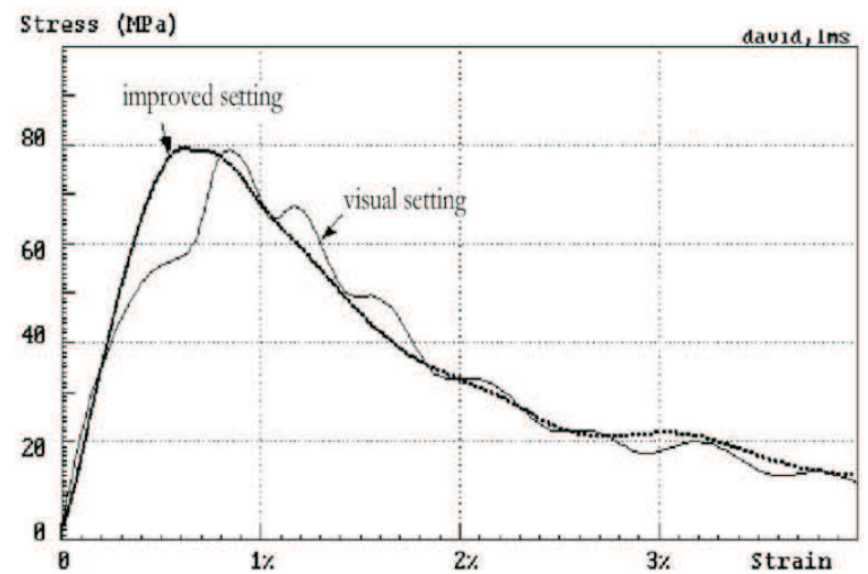

Fig. 15. an example of possible effect of the method. Case of a concrete specimen. 
Recall that the method does not work correctly without the dispersion correction and that it relies on an initial non negligible elastic behaviour of the specimen.

It still perfectly works with (slightly) viscoelastic bars (as Nylon or PMMA bars) but not with a purely viscous specimen.

\subsection{Punching of the bar-end by the specimen}

After using this method during years, we have observed that the expected modulus of the specimen was found when the specimen diameter was close to that of the bars. The measured value was lower than the expected one in most situations when the specimen diameter was smaller than that of the bars. Furthermore, for a given specimen diameter, the discrepancy was decreasing with specimen length.

This problem, due to the local punching of the bar end by the specimen, has been addressed by Safa \& Gary (2010). They have shown that this effect is like that of an added spring at the end of the bars: the displacement due to punching is proportional to the applied (and measured) force. A simple formula has been established that gives the value of the displacement due to punching. Results are summarised in figure 16 next page.

Note that the 1-D elastic behaviour of punching allows for taking account of its contribution in the elastic simulation process. Consequently, the Young's modulus of any material specimen can be directly estimated by means of the elastic simulation process.

It is clear that the relative influence of the displacement due to punching increases when the length of the specimen decreases. This is especially important at high or very high strain-rates that need shorter specimen (as the incident striker-speed is limited by the care of the gauges) when the specimen diameter is under the half of that of the bar.

For a real test made at $10000 / \mathrm{s}$, with a specimen metricconverterProductID3 $\mathrm{mm} 3 \mathrm{~mm}$ thick, diameter $6.5 \mathrm{~mm}$ (steel bars, $12 \mathrm{~mm}$, striker speed $25 \mathrm{~m} / \mathrm{s}$ ), the equivalent in strain due to punching, at the plastic plateau, is around $14 \%$. On the other side, at low strain rates, when the total measured strain is small, the effect of punching is still important as illustrated in figure 17 . 


\section{Summary of main results for SHPB}

We consider a classical SHPB apparatus where input and output bars are identical with diameter $D$ and with $\beta, v$ and $E$ the density, Poisson ratio and Young's modulus, respectively, of their material. The geometrical characteristics of the specimen are $d_{*} l_{n}$ and $S_{*}$, corresponding to its diameter, length and cross sectional area, respectively.

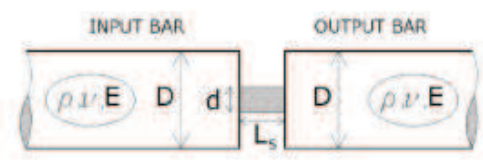

At any time throughout the experiment, the strain of the sample is obtained as

$$
\epsilon(t)=\epsilon_{\text {sHPv }}(t)-\epsilon_{\operatorname{panat}}(t)
$$

where

- $\epsilon_{\operatorname{manh}}(t)=2 K_{p} \frac{\sigma_{S H P B}(t) S_{s}}{l_{s}}$

- $\operatorname{sHPB}(t)$ and $\sigma_{S H P B}(t)$ are the strain and stress, respectively, obtained by standard SHPB formulas.

- $K_{p}=\frac{16}{3 \pi^{2}} \frac{1-v^{2}}{d E} H_{p}\left(\frac{d}{D}\right)$

$$
\begin{aligned}
& H_{p}(x)=2-\left(x+\frac{1}{x}\right) E(x)-\left(x-\frac{1}{x}\right) h(x) \\
& E(x)=\int_{0}^{4} \sqrt{1-x^{2} \sin ^{2} \theta} d \theta, K(x)=\int_{0}^{\frac{2}{2}} \frac{d \theta}{\sqrt{1-x^{2} \sin ^{2} \theta}}
\end{aligned}
$$

Tabulated results for the function $H_{p}(x)$ are given in the table underneath. Values for $0.5<\mathrm{x} \leq 1$ are obtained by linear interpolation.

\begin{tabular}{|c|c|c|c|c|c|c|c|c|c|c|c|c|c|c|}
\hline $\mathrm{X}$ & 0.10 & 0.15 & 0.20 & 0.25 & 0.30 & 0.35 & 0.40 & 0.45 & 0.50 & 0.60 & 0.30 & 0.80 & 0.90 & 1.00 \\
\hline $\mathrm{H}_{4}(\mathrm{x})$ & 1.765 & 1.648 & 1.531 & 1.416 & 1.301 & 1.188 & 1.076 & 0.967 & 0.860 & 0.088 & 0.516 & 0.344 & 0.172 & $a$ \\
\hline
\end{tabular}

Fig. 16. Summary of punching data for SHPB. 


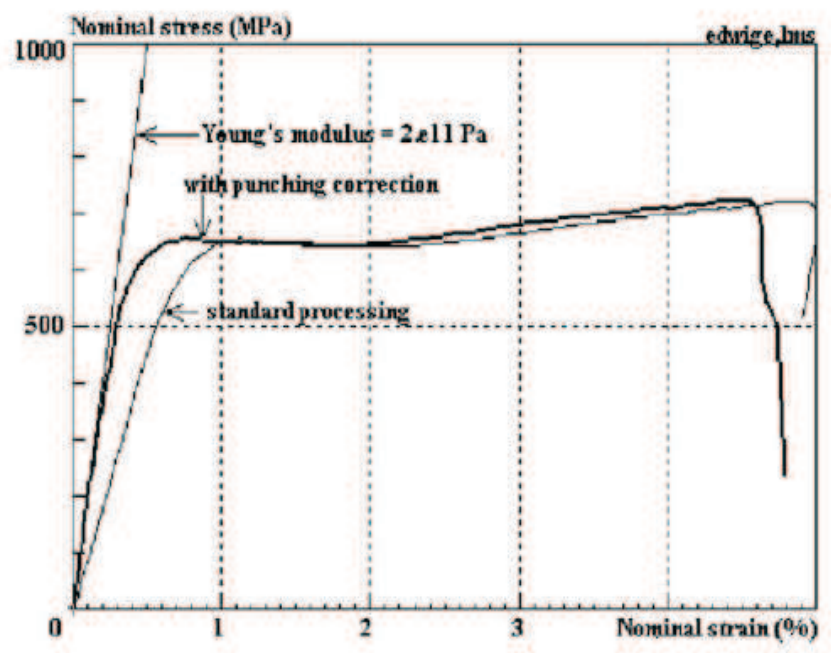

Fig. 17. Influence of the punching correction. (20 mm steel bar, striker speed $2.79 \mathrm{~m} / \mathrm{s}$, strain-rate around $100 / \mathrm{s}$ ).

\subsection{Force matching. Critical case of the input side.}

This problem has nothing to do with a so called "impedance matching" problem (discussed later).

In most cases, especially when testing metallic specimens, input and output forces are almost equal. This is not always the case, as shown later. Anyway, in the following analysis, we use this assumption to make clearer the "force matching" problem.

Considering formula (9), it appears that a correct measurement of the output force is only possible when the measured output strain is itself significant. It can be considered that strain measurements are done with a precision around $5.10^{-6}$ when using classical strain-gauges. With semiconductor gauges, it can be improved by a factor 50 . Considering that an acceptable relative precision of $2 \%$ is suitable for any measurement, the maximum strain measured should be more than $2,5 \cdot 10^{-4}$. Using formula (9) allows for the calculation of the minimum suitable output force.

In the example of a steel bar (20 mm diameter), this force is around 15 $\mathrm{kN}$. The specimen area being at maximum equal to the bar diameter, the corresponding stress is $50 \mathrm{MPa}$. For weaker materials, it is necessary to use semiconductor gauges or a better amplifying system (or a bar with a low Young's modulus, as it is the case of most viscoelastic bars).

Let us then assume that a material, with a maximum stress of $50 \mathrm{MPa}$, is tested with the steel bar, very carefully. Considering now formula (9) for 
the input force, it appears that a precise measurement is not possible when the amplitude of the incident wave and the opposite of the amplitude of the reflected wave are too close.

Keeping this example, if we wish to obtain high strain rates, we use a small specimen length. For sake of simplicity, we assume that the tested material is not strongly rate sensitive. A simple simulation, with a specimen $5 \mathrm{~mm}$ long, shows that the striker speed must be $15 \mathrm{~m} / \mathrm{s}$ to obtain an average strain rate close to $3000 / \mathrm{s}$. In this case, the absolute amplitude of the reflected wave only differs by $15 \%$ from the amplitude of the incident wave. A precise calculation of the input force with formula (9) is then difficult.

If we consider that an acceptable result is obtained when both waves differ by more than $20 \%$, the maximum strain rate giving an acceptable result is, for this example, around $400 / \mathrm{s}$. It can be concluded that materials with a plateau stress under $50 \mathrm{MPa}$ cannot be safely tested with $20 \mathrm{~mm}$ steel bars at strain-rates over $400 / \mathrm{s}$.

More generally speaking, weak materials need the use of soft bars.

This problem should be called the "force-matching" problem as it has nothing to do with the specimen impedance, but only with the product of the Young's modulus of the bar by its area to be compared with the maximum force to be measured.

Optimizing specimen dimensions. In order to insure good measurements on the input side (more often of the force) the specimen has to be design depending on the required strain-rate. The problem is also to choose the appropriate striker speed to obtain the strain rate. One can use a method presented by CityplaceGary (2001), which is based on simple assumptions (one-dimensional wave analysis, specimen equilibrium, evaluation of the material stress: say $\sigma_{y}$ ). The amplitude of the incident wave is deduced from the striker speed V (eq. 11a). The amplitude of the transmitted wave is deduced from the specimen section $S_{s}$ and $\sigma_{y}$ (eq. 11b), and the amplitude of the reflected wave is deduced from the equilibrium (eq. 11c).

$\varepsilon_{i}=\frac{V}{2 c}(11 \mathrm{a}), \varepsilon_{t}=\frac{S_{s} \sigma_{y}}{S_{b} E_{b}}(11 \mathrm{~b})$,

$\varepsilon_{r}=-\varepsilon_{i}+\varepsilon_{t}(11 \mathrm{c}), \dot{\varepsilon}_{s}=\frac{2 c \varepsilon_{r}}{l_{s}}(11 \mathrm{~d})$

where $S b$ and $E b$ are the section and the Young's modulus of the bars, respectively.

The average strain rate is now given by formula $(11 \mathrm{~d})$, where $c$ is the wave speed in the bars and $l s$ is the specimen length. It is then possible to make sure that the relative amplitude of the reflected wave is small enough in comparison with the relative amplitude of the incident wave. If we look at formulas (8) and (9), it can be seen that the best situation, i.e., that giving the most accurate force and speed results, is that occurring when the amplitudes of the transmitted and reflected waves are fairly similar. 
These formulas make it possible to easily determine optimized specimen dimensions and striker speeds.

\subsection{Impedance matching}

The impedance matching problem, has been addressed by Davies and Hunter (1963). It mainly deals with the homogeneity of stresses and strains in the specimen, during a Hopkinson bar test, and especially at early instants.

During this loading phase, input and output forces are alternatively bigger than the other. An example is shown in fig 18, from Klepaczko (2007) figure 3.11a, p 206. The gap between both forces decreases when the speed of waves in the specimen increases and when its length decreases. It also decreases when the amplitude in force (in stress in the present figure) of the first step decreases.

Here is the "impedance" matching problem that describes the conditions to make this step small enough. Furthermore, this step is softened if the rising time of the loading is increased. This point is one reason for using pulse shapers.

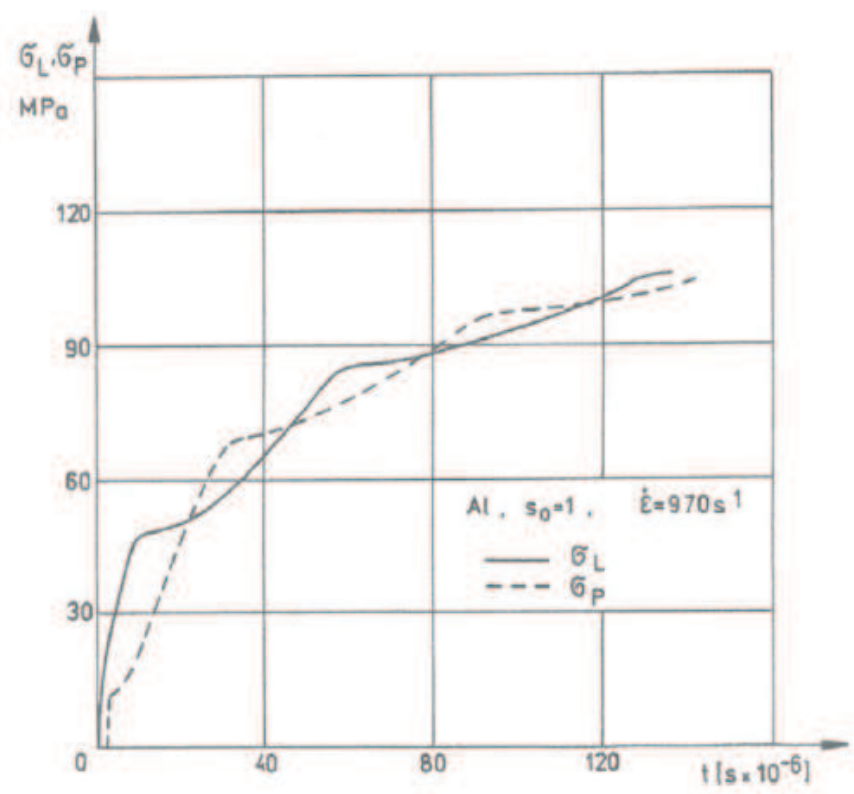

Fig. 18. Elastic input and output stresses at early instant of the loading from Klepaczko (2007). 


\subsection{Holding the specimen: dealing with one-point measurements}

Measurements with bars are always analyzed within the 1-D hypothesis (even with dispersion and punching corrections as both are only expressed as a function of the axial coordinate of the bar). In other words, force and speed measurements, on each side, are made at a single point. This basic hypothesis does not appear when Kolsky's formulas are used for a direct stress-strain relation. It is then important to keep in mind this aspect, especially in situations where holding the specimen leads to the use of devices that induce an impedance change along the wave propagation path.

Some examples are given here.

When compression bars are used to test a material specimen, the specimen diameter must be smaller than the diameter of the bar to ensure a quasi-uniaxial state of stress. When very weak material are tested, even with viscoelastic bars, the quality of the measurement is limited by force matching problems described above.

In order to overcome this limitation, one could use at bar ends a device with a larger diameter and then be able to hold a specimen with a much larger specimen. Such a technique is illustrated in figures 19 and 20.

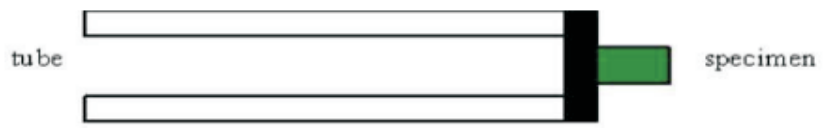

Fig. 19. Impedance change between the bar (tube) and the specimen.

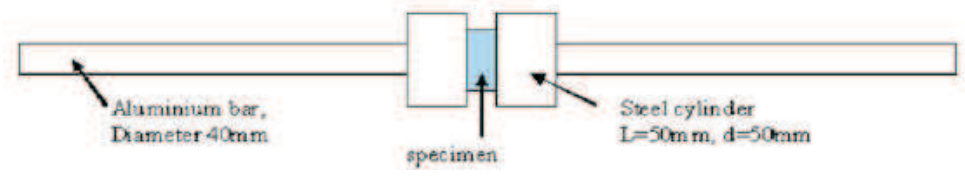

Fig. 20. Special device for a larger specimen.

The first question here is to decide at which point is made the measurement: end of the bar or specimen face?

In order to illustrate this problem, a brass specimen has been tested in the situation shown in fig. 20 and in a classical situation with the same (aluminium) bars. The corresponding recorded waves are shown in fig. 21 


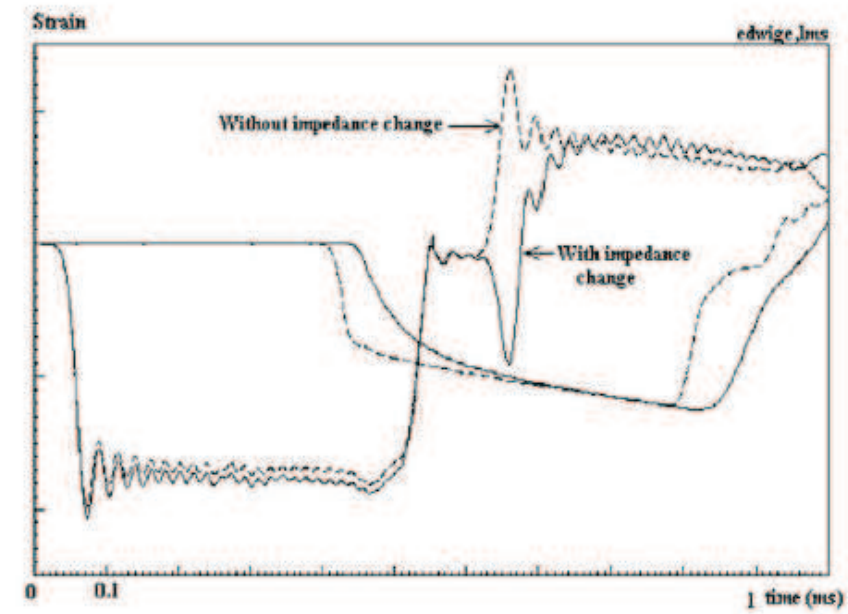

Fig. 21. Illustration of the influence of a matching device - waves. It is observed that the strongest influence appears in the reflected wave, and that the transmitted wave is smoothed (this is not a general results as it depends on each impedance ratio device/bar).

A standard processing of the waves will then give wrong results when matching devices are used, as shown in figures 22 and 23.

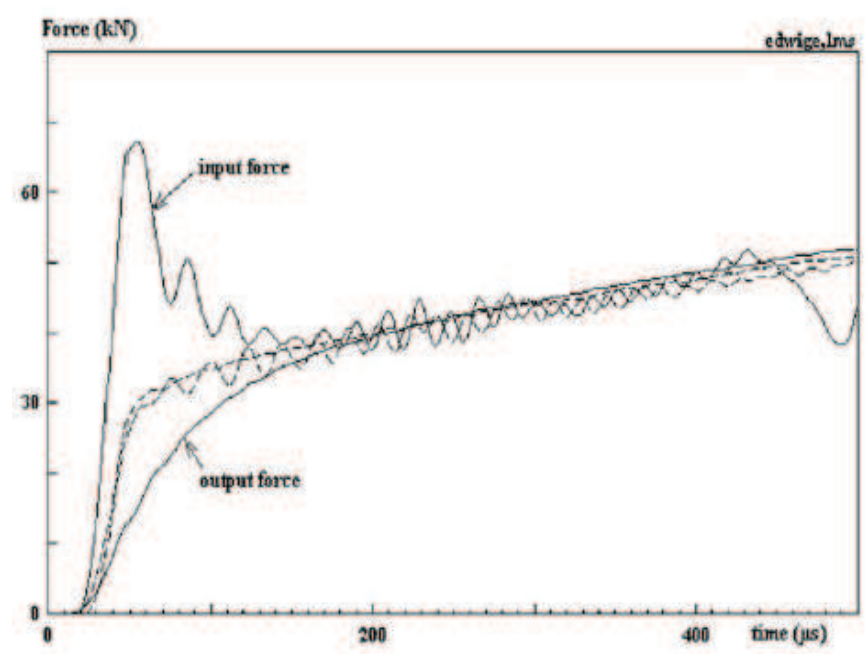

Fig. 22. Apparent equilibrium with and without devices. 


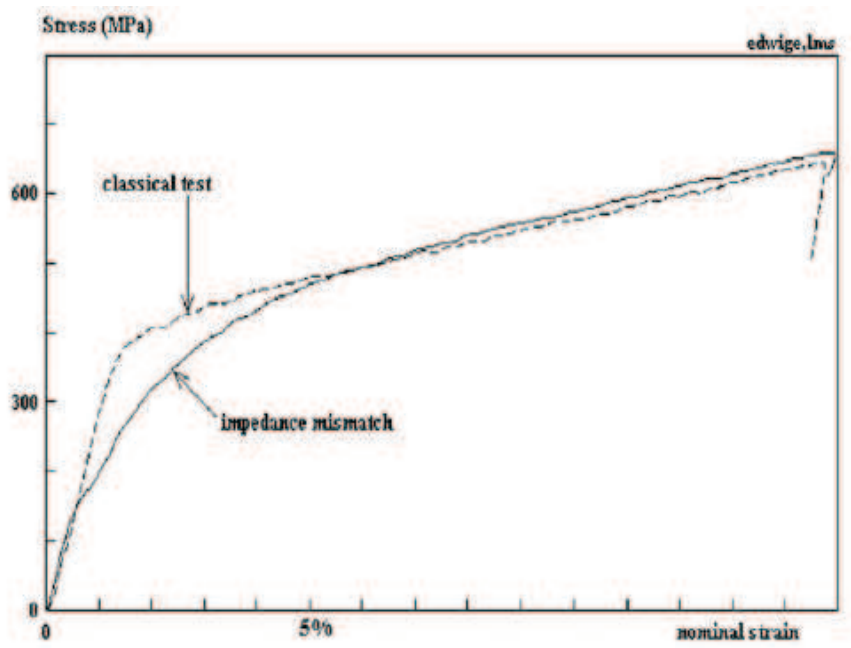

Fig. 23. Apparent stress-strain relation with and without devices.

Looking at the stress-strain diagram shows a difference that is not so large, in particular for larger strains. The main effect is a smoothing of the stress-strain relation that prevents for an acceptable evaluation of the elastic limit of the tested material.

When it is not possible to avoid the use of a matching device, an impedance correction is possible. A simple way is to use a one dimensional analysis. Knowing the waves at the end of the bar (in the part with a constant impedance), the waves at specimen faces can be calculated, assuming the 1-D elastic behaviour of the devices of known impedance. The forces and the displacements applied to the specimen faces are then deduced.

\subsection{Undetermined specimen ends}

A good example is found in a work of Gary and Nowacki (1994). They proposed a device to convert compression in shear. The idea is summarised in fig. 24.

The (say) input hollow cylinder has the same impedance than the input bar when the output cylinder has the same impedance as the output bar. The problem here is that the beginning and the end of the specimen are not defined. Consequently one has to consider that the shear-displacement curve is filtered with a cut-off frequency depending on the length of the shear zone. 


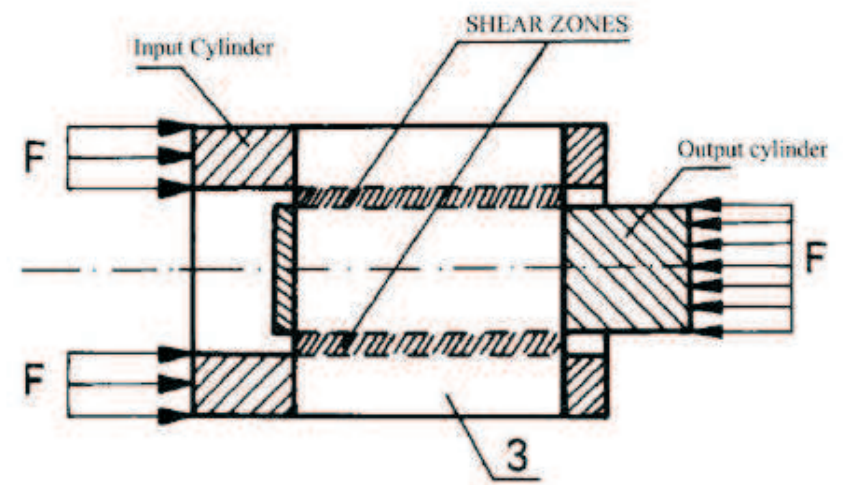

Fig. 24. Conversion compression to shear.

\section{Specimen behaviour}

The Split Hopkinson pressure bar arrangement can give very accurate measurements of forces and velocities at both sample faces if the data processing is carefully performed. Relating material properties to measurements of forces and velocities at the two specimen faces is a completely independent problem.

For this reason, we have distinguished the problems of measuring accuracy of SHPB and material property identification from the measured data.

\subsection{Axial homogeneity}

Due to the presence of waves in dynamic experiments, both the stress and strain fields within a specimen are seldom uniform. A dynamic material test should be designed such as to minimize this inherent non-uniformity, a condition which is typically associated with "quasi-static equilibrium". However, when testing purely elastic materials such as fiber reinforced composites or low impedance materials, the validity of this assumption needs to be checked with care. Note that there are situations where force equilibrium can exist without strain homogeneity as it is often the case with structural materials like honey-comb or foam.

Before computers became generally available, the assumption of quasistatic equilibrium of the specimen had a special importance from a data processing point of view.

The historical point is that, in the 50's, signals could not be recorded. 
The idea was to associate, in a "memory scope" (the image was stored in an analogic way) the "strain" and the "stress" signals and directly observe a curve proportional to the stress-strain curve of the material. First, the strain-rate was analogically integrated with respect to time. Then, the simplest way to synchronize both signals was to produce them at the same time. This was the reason to use both bars of same length with each gauge in the middle.

With the use of modern numerical acquisition, the output gage can now be positioned closer to the specimen interface; consequently, the output bar can be shorter than the input one (and there is no good reason to choose its length equal to that of the input bar). The use of "equilibrium" condition was also crucial at that time as the input force could not be directly measured.

Optimal design with equilibrium. It is worth noting here that this equilibrium condition could be used with different input and output bars leading to slightly more complex formulas. In the same way, other formulas could be derived using any pair of waves (among three).

Let's consider the idea of using only the incident and transmitted waves. For sake of simplicity we suppose that the two bars are still identical but the results could be generalized to a SHPB made of two different bars. Assuming equilibrium, formula 3 can also be written:

$$
\varepsilon_{s}(t)=\int_{0}^{t} \dot{\varepsilon}_{s}(\tau) d \tau=-\frac{2 c_{b}}{l_{s}} \int_{0}\left(\varepsilon_{i}(\tau)-\varepsilon_{t}(\tau)\right) d \tau \quad \sigma_{s}(t)=\frac{A_{b}}{A_{s}} E_{b} \varepsilon_{t}(t)
$$

where $A_{b}$ is the area of the bar, $E_{b}$ its Young's modulus and $A_{s}$ the area of the specimen.

It can be seen that only the incident and the transmitted waves are now involved in the processing formulas. Finding an optimal position for the gauges, close to the input side of the bars where the waves arrive first, (see the case of direct impact) will then lead to an increased measured strain. It is then easy to find the optimal design close to the one shown in figure 25 . The bars have now to have the same length to allow for the same duration of the incident and the transmitted waves. Consequently, the striker has to be as long (or longer) as the remaining distance between the gauges and the end of the bars. 


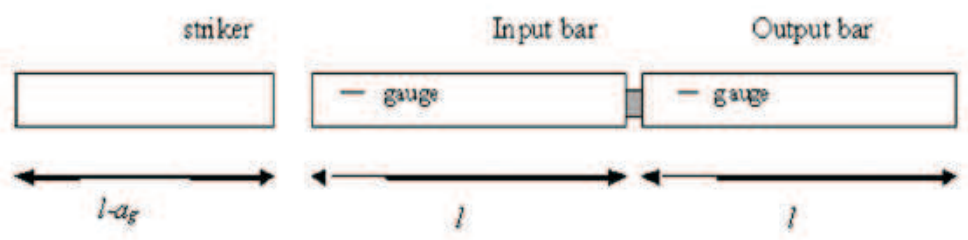

Fig. 25. With equilibrium, a configuration for an increased duration measurement.

Evaluation of specimen behaviour. The evidence of non equilibrium is found in early instants of the loading, when the first wave in the specimen has not yet reached its end. At this instant the input force exists when the output force is still null. Knowing that specimen equilibrium is never achieved exactly, we seek the best of the commonly used stress-strain curve estimates in a SHPB experiment. This question is studied by Mohr et al. (2010). The time shift of the waves is found to play a critical role as far as the accuracy is concerned.

More specifically, it is found that the omission of artificial time shifts (as illustrated in fig 26) provides the best stress-strain curve estimates. In other words, once the force and displacement histories are known at the specimen boundaries, accurate estimates of the stress-strain curves should be made without further shifting the signals on the time axis.

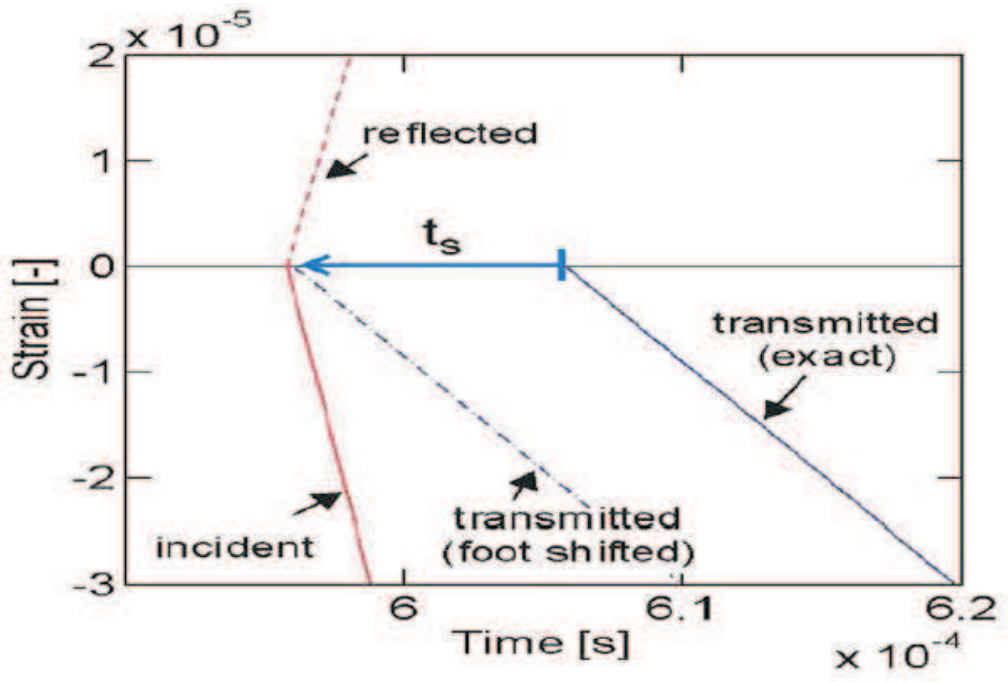


Fig. 26. Exact theoretical waves as shifted at specimen faces. The dashed blue line shows the strain history of the transmitted wave after shifting the beginning of this wave in time (so-called "foot shifting") such that all strain histories begin simultaneously.

Estimations evaluated by Mohr et al. (2010) are based on exact explicit calculation done in the frequency domain. Results confirm that the error (for all formulas) increase with the impedance mismatch between the specimen and the bars. It is emphasized that, in standard testing situations, exact shifting (illustrated in fig 26) is much more important than strain or force approximation (with 2 or 3 waves).

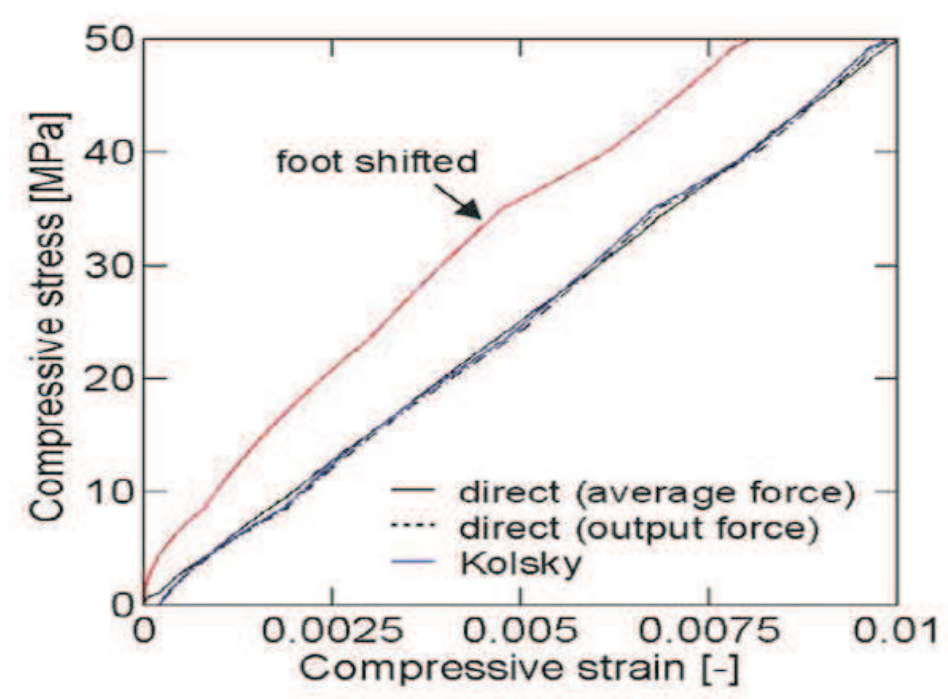

Fig. 27. Plot of the estimated stress strain curves for a dynamic compression experiment on PMMA (steel bars metricconverterProductID20 $\mathrm{mm} 20 \mathrm{~mm}$ ).

The estimates which are based on the force and displacement time histories at the specimen boundaries without artificial time shifts, provide the most accurate stress-strain curve. Unless accurate input force measurements are available, the combination of the average strain with the output force based stress estimate is recommended for standard SHPB experiments. An illustration is given in fig. 27.

Recall that in the elastic range and in standard SHPB testing, when the specimen diameter is smaller than half of that of the bars, the incidence 
of the punching correction on the quality of the results is the most important.

\subsection{Radial homogeneity. Friction and inertia}

We must keep in mind that, alike in all testing situations, axial stress and strain homogeneity (considered up to now) is not the only one to be considered. In quasi-static testing, it is possible to directly measure the strain in a "gauge section" of the specimen where 1-D assumptions are well verified. In SHPB, when using a cylindrical specimen, it is more difficult to avoid friction at specimen faces. This friction prevents the expected radial expansion quantified by Poisson's ratio.

The consequence is that the triaxial stress-state is not uniform along the axial loading. It induces an average over-stress in the common situation of elastoplastic materials.

This radial expansion is also prevented by inertia effects that induce lateral confinement.

An empirical formula has been established by Malinowski and Klepaczko, (1986) that takes account of both effects. This formula is valid for purely plastic materials but gives a first order correction that applies to many other materials.

$$
\sigma-\sigma_{0}=\frac{\mu \sigma}{3 s}+\frac{\rho d^{2}}{12}\left(s^{2}-\frac{3}{16}\right)\left(\dot{\varepsilon}^{2}+\ddot{\varepsilon}\right)+\frac{3 \rho d^{2}}{64} \ddot{\varepsilon}
$$

where, $\sigma_{0}$ : ideal value, $\sigma$ : actual value (true stresses)

land dactual values of length and specimen diameter and $s=l / d$

$\rho$, density assumed to be constant

$\mu$ friction Coulomb coefficient (usually $<0.1$ ).

This formula indicates that the inertia effect is much smaller than that of friction for common metal testing (bar diameter $<20 \mathrm{~mm}$ ). It becomes significant for bigger specimens $(>30 \mathrm{~mm})$ like found for rock or concrete testing when big specimen sizes are required for a good average material representation. It is seen in this case that some artificial strain rate effects can be attributed to bi-axial loading induced by inertial confinement.

In the case of testing where a quasi-static radial confinement is added see Gary and Bailly (1998), and Forquin et al. (2010) - inertia effects are prevented and do not operate anymore.

\subsection{Adiabatic heating}

The duration of a dynamic compression test with SHPB is almost always under $1 \mathrm{~ms}$. For many materials, it induces large strains (depending on strain-rate) that produce work which is (partly ?) converted into heat. In 
case of metal or polymer testing, it can induce a specimen temperature increase in the range up to $100^{\circ} \mathrm{C}$.

It is then important to account for this temperature increase for an improved evaluation of the material behaviour. This question has been investigated by various authors who have proposed techniques for high speed temperature measurements (more often based on infrared specimen emission). See for instance Rittel (1999) and Negreanu et al. (2009).

\subsection{Inverse methods}

On the basis of force and velocities measurements at specimen faces, an approach of the specimen behaviour based on an inverse method is theoretically possible, as shown by Rota (1994), as these four values are superabundant measurements.

3-D dynamic numerical simulation could allow, in this case, accounting for friction, radial inertia and possibly temperature increase. If an appropriate form of the material behaviour with some parameters to be determined is known, using a part of data (two velocities, for example) as the input data, another part of data (the two forces) associated with the given parameters can be calculated. The best set of parameter which gives the calculated forces well in agreement with the measured ones can theoretically be found.

In order to further investigate this approach, we shall limit the analysis to axial 1-D effects by means of a fast 1-D transient simulation based on the Sokolovsky (1948) and Malvern (1951) approach.

The uniaxial governing equation and the constitutive law are written in equations (12)

$$
\begin{gathered}
\frac{\partial \sigma(x, t)}{\partial x}=\rho \frac{\partial \mathrm{v}(x, t)}{\partial t} \\
\frac{\partial \varepsilon(x, t)}{\partial t}=\frac{\partial \mathrm{v}(x, t)}{\partial x} \\
f(\sigma, \varepsilon, \dot{\sigma}, \dot{\varepsilon}, \ldots)=0
\end{gathered}
$$

where $\sigma, \varepsilon, \mathrm{v}$ are the stress, the strain, and the particle velocity in the specimen. $\rho$ is the mass density.

The boundary conditions at the two faces of the specimen are given as follows:

$$
\begin{gathered}
\sigma(x, t)-\frac{E_{B} S_{B}}{C_{B} S_{S}} \mathrm{v}(x, t)=2 \frac{S_{B}}{S_{S}} E_{B} \varepsilon_{i}(t) \quad \text { at the input side } \\
\sigma(x, t)+\frac{E_{B} S_{B}}{C_{B} S_{S}} \mathrm{v}(x, t)=0 \quad \text { at the output side }
\end{gathered}
$$


$E_{B}, C_{B}, S_{B}, S_{s}$ denote the Young's modulus, wave velocity, cross-sectional area of the bar and the section of the specimen.

Once the specimen behaviour is assumed, the direct problem described by equations $(12,13)$ corresponds to an uni-dimensional SHPB simulation. As mentioned above, the simulation would be just the preliminary stage of the inverse problem which consists in finding the parameters of the model. The inverse methods need fast calculation procedures to allow for a great number of direct calculations with different sets of parameters if necessary. For this purpose, the specimen behaviour should be numerically easy to calculate. This is why it is chosen to use a Sokolovsky-Malvern model type for the specimen, (14).

$$
\begin{aligned}
& \frac{\partial \varepsilon}{\partial t}=\frac{1}{E} \frac{\partial \sigma}{\partial t} \quad \text { if } \quad \sigma \leq \sigma_{s} \\
& \frac{\partial \varepsilon}{\partial t}=\frac{1}{E} \frac{\partial \sigma}{\partial t}+g(\sigma, \varepsilon) \quad \text { if } \quad \sigma>\sigma_{s}
\end{aligned}
$$

The characteristic network in this case is composed of families of straight lines so that the numerical integration of equation $(13,14)$ by the method of characteristics is very efficient. In comparing the given behaviour with the average stress strain curve obtained from three simulated waves, the efficiency of classical SHPB analysis for this type of materials can be evaluated.

It is noted that the Sokolovsky-Malvern constitutive model is a quite general rate-sensitive one. Though it has been introduced initially for metals, it can be also used to describe the non-metallic materials if the function $g(\sigma, \varepsilon)$ is correctly chosen -see Critescu (1967).

In order to have a general idea of the efficiency of the classical SHPB analysis for this type of constitutive models, a popular rheological model (Fig. 28) is examined here, which is of Sokolovsky-Malvern type with the $g(\sigma, \varepsilon)$ expressed as follows,

$$
g(\sigma, \varepsilon)=\frac{\left(1+\frac{E_{v}}{E}\right) \sigma-E_{v} \varepsilon}{\eta}
$$

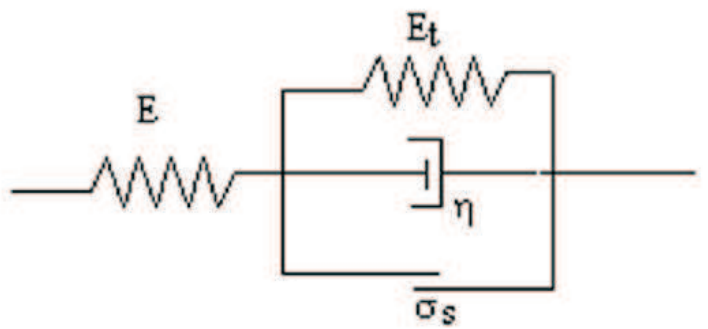


Fig. 28. Rheological model for 1-D standard elasto-visco-plasticity.

A number of simulations were done for different sets of parameters. It has been found, like for previous results on metals, that the classical average stress-strain curve is in good agreement with the curve given by SHPB standard analysis at relative important strains. However, in the range of small strains, it is acceptable when the viscosity is low even if there is no axial uniformity. On the other hand, when the viscosity is high, the average (SHPB) curve is quite far from the given behaviour.

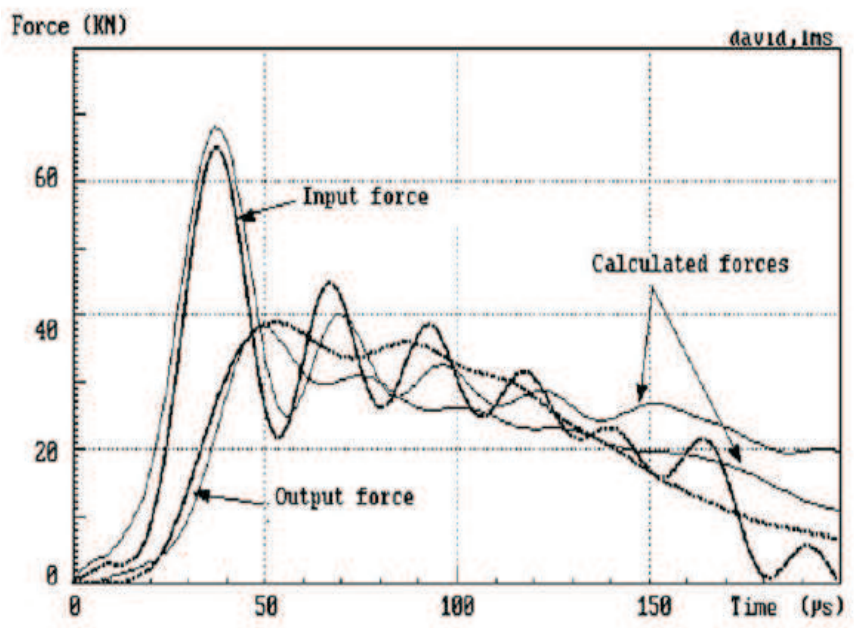

Fig. 29. Simulated and measured forces for rock-salt. 


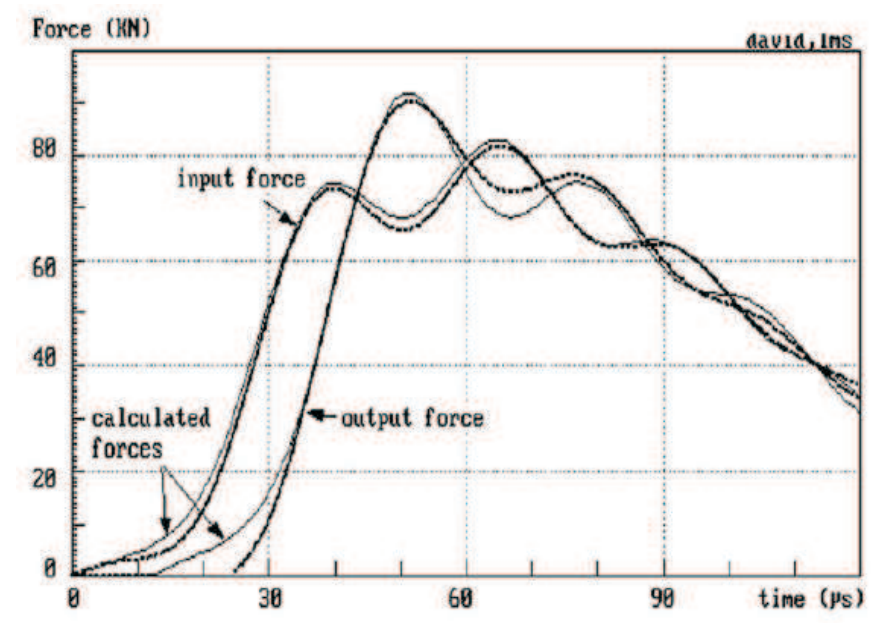

Fig. 30 Simulated and measured forces for concrete.

An illustration of the method is presented in two particular cases: for salt in fig. (29) which exhibits a strong viscoplastic behaviour and for concrete fig. (30) which breaks, showing a negative strain hardening, at small strains. In both cases, equilibrium conditions are obviously not satisfied.

The chosen model with the set of parameters which gives the best agreement can be considered as a representative model of the specimen in this test. As a result, the stress and strain fields in the specimen are evaluated so that a stress-strain curve is found. The assumption on the uniformity of stress and strain fields is then not needed in such a method. The only hypothesis used is that specimen tested manifests the same properties everywhere. Furthermore, it is possible to give a stress strain curve at a constant strain rate via the model and the set of parameters identified.

This stress-strain curve can then be compared with the one obtained with the classical SHPB analysis. For the test on salt, the two curves are quite far from each other in the range of small strains. The inverse calculation is then in this case the only way to obtain an accurate result for this type of materials. On the other hand, for the test of concrete, the average stressstrain curve is rather close to the curve derived from the model, as a kind of averaging operates between input and output forces. The inverse calculation could then be avoided. 


\section{Measurement of the dispersion relations}

A method is briefly presented here that provides a very accurate estimation of the dispersion relations. One reason is that it is a one point method; the other is that it is based on simple formulas and an easy identification of the wave speed and damping at a given finite set of frequencies.

Furthermore, it allows for a subsequent mathematical analysis which includes noise reduction and provides a rheological model with a number of elements that is in accordance with the complexity of the material.

\subsection{Impact test method. Theory}

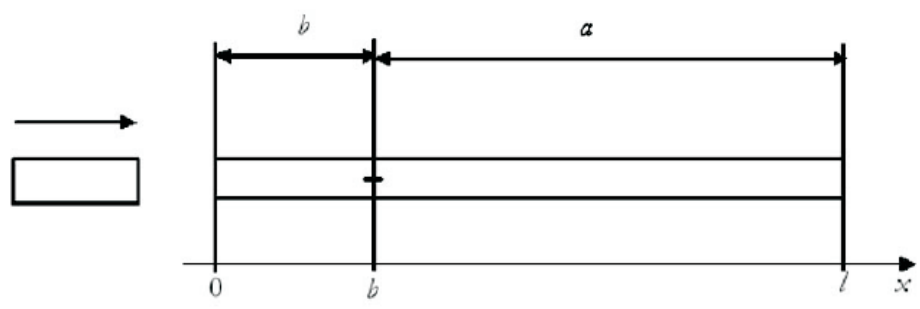

Fig. 31 Impact test with a uniform viscoelastic bar specimen.

At one end, $x=0$, the bar is impacted axially by a striker that separates from the bar after the generation of a compressive primary pulse in the bar. The other end of the bar, $x=l$, is free. The strain $\varepsilon_{b}(t)=\varepsilon(b, t)$ is recorded at a distance $a$ from the free end and $b$ from the impacted end $(a+b=l)$. The primary pulse should be shorter than $2 a$ so that there is no overlap in the measured strain of this pulse and the first pulse reflected from the free end. However, it should be much longer than the diameter of the bar so that approximate 1D conditions prevail - Hillstrm et al (2000).

In the frequency domain, the strain in the bar can be expressed as:

$$
\hat{\varepsilon}(x, \omega)=A(\omega) e^{-\mathrm{i} \xi(\omega) x}+B(\omega) e^{\mathrm{i} \xi(\omega) x},
$$

where

$$
\xi^{2}(\omega)=\rho \omega^{2} / E(\omega), \xi(\omega)=k(\omega)-\mathrm{i} \alpha(\omega)
$$

Here, $\hat{\varepsilon}(x, \omega)$ is the Fourier transform of $\varepsilon(x, t)$, and $A(\omega)$ and $B(\omega)$ are complex amplitudes of waves travelling in the directions of increasing and decreasing $x$, respectively, $k(\omega)$ is the wave number and $\alpha(\omega)$ is the damping coefficient.

In order to determine an expression for the recorded strain $\hat{\varepsilon}_{b}^{1}(\omega)=$ $\hat{\varepsilon}(b, \omega)$ associated with the primary pulse alone, the amplitudes $A$ and $B$ are 
first determined from Eq. (16) and the boundary conditions $\hat{\varepsilon}(0, \omega)=\hat{\varepsilon}_{0}(\omega)$ and $B(\omega)=0$ for a semi-infinite bar $x \geq 0$, where $\hat{\varepsilon}_{0}(\omega)$ is the strain at the impacted end. With these amplitudes and $x=b$ inserted, Eq. (16) gives

$$
\hat{\varepsilon}_{b}^{1}(\omega)=e^{-i \xi b} \hat{\varepsilon}_{0}(\omega)
$$

The recorded strain $\hat{\varepsilon}_{b}^{1}(\omega)=\hat{\varepsilon}(b, \omega)$ associated with the complete train of pulses is determined similarly. With amplitudes $A$ and $B$, and $x=b$ and $l-b=a$ inserted, Eq. (16) gives

$$
\hat{\varepsilon}_{b}^{\infty}(\omega)=\frac{\sin (\xi a)}{\sin (\xi l)} \hat{\varepsilon}_{0}(\omega) .
$$

Dividing the members of Eq. (19) by those of Eq. (18) eliminates the strain $\hat{\varepsilon}_{0}(\omega)$ at the impacted end which normally cannot be measured. The elimination of this strain makes the difference between the method used here and that used by Othman et al. (2001). Substituting $\xi$ from the second of Eqs. (17) into the result, one gets the ratio $\hat{\varepsilon}_{b}^{\infty}(\omega) / \hat{\varepsilon}_{b}^{1}(\omega)$ which gives

$$
\left|\hat{\varepsilon}_{b}^{\infty}(\omega)\right|^{2}=\left|\hat{\varepsilon}_{b}^{1}(\omega)\right|^{2} e^{2 \alpha b} \frac{\sin ^{2}(k a)+\sinh ^{2}(\alpha a)}{\sin ^{2}(k l)+\sinh ^{2}(\alpha l)} .
$$

Resonance occurs at the angular frequencies $\omega=\omega_{m}, \mathrm{~m}=1,2, \ldots$ which correspond to the wave numbers, wavelengths and phase velocities

$$
k_{m}=\frac{m \pi}{l}, \lambda_{m}=\frac{2 \pi}{k_{m}}=\frac{2 l}{m}, c_{m}=\frac{\omega_{m}}{k_{m}}=\frac{\omega_{m} l}{m \pi},
$$

respectively.

It is assumed that within the $m$ :th resonance peak $\alpha=\alpha_{m} \omega / \omega_{m}$ can be taken as directly proportional to angular frequency and $c(\omega)=\omega / k(\omega)=$ $c_{m}$ can be taken as constant. By use of the third of Eqs. (21) we then obtain the relation between wave number and angular frequency within the resonance peak. Inserting these expressions for $\alpha(\omega)$ and $k(\omega)$ into Eq. (20) we get $(m=1,2, \ldots)$

$$
\left|\hat{\varepsilon}_{b}^{\infty}(\omega)\right|^{2}=\left|\hat{\varepsilon}_{b}^{1}(\omega)\right|^{2} e^{2 \alpha_{m} b \omega / \omega_{m}} \frac{\sin ^{2}\left(k_{m} a \omega / \omega_{m}\right)+\sinh ^{2}\left(\alpha_{m} a \omega / \omega_{m}\right)}{\sin ^{2}\left(k_{m} l \omega / \omega_{m}\right)+\sinh ^{2}\left(\alpha_{m} l \omega / \omega_{m}\right)}
$$

within the $m: t h$ resonance peak. In this relation, the spectra $\left|\hat{\varepsilon}_{b}^{\infty}(\omega)\right|^{2}$ and $\left|\hat{\varepsilon}_{b}^{1}(\omega)\right|^{2}$ can be determined experimentally.

For each resonance $\mathrm{m}=1,2, \ldots$, the resonance frequency $\omega_{m}$ and damping coefficient $\alpha_{m}$ can be estimated by minimizing the difference between the 
resonance peaks represented by the left and right members of Eq. (16). From $\omega_{m}$ and $m$, the complex modulus $E_{m}$ at each resonance frequency can be finally obtained as

$$
E_{m}=\rho\left(\frac{\omega_{m}}{\xi_{m}}\right)^{2}, \xi_{m}=\frac{m \pi}{l}-\mathrm{i} \alpha_{m}, m=1,2, \ldots
$$

\subsection{Experimental set-up and procedure}

Impact tests were carried out with a Nylon bar specimen of length 3045 $\mathrm{mm}$, diameter $10.2 \mathrm{~mm}$, and density $1149 \mathrm{~kg} / \mathrm{m}$. The striker had length $174 \mathrm{~mm}$ and the same diameter $10.2 \mathrm{~mm}$ and material as the bar, and its impact velocity was $3.7 \mathrm{~m} / \mathrm{s}$. The strain was recorded with $500 \mathrm{kHz}$ sampling frequency, and the signal vanished completely before the end of the recording window (fig. 32 and 33)

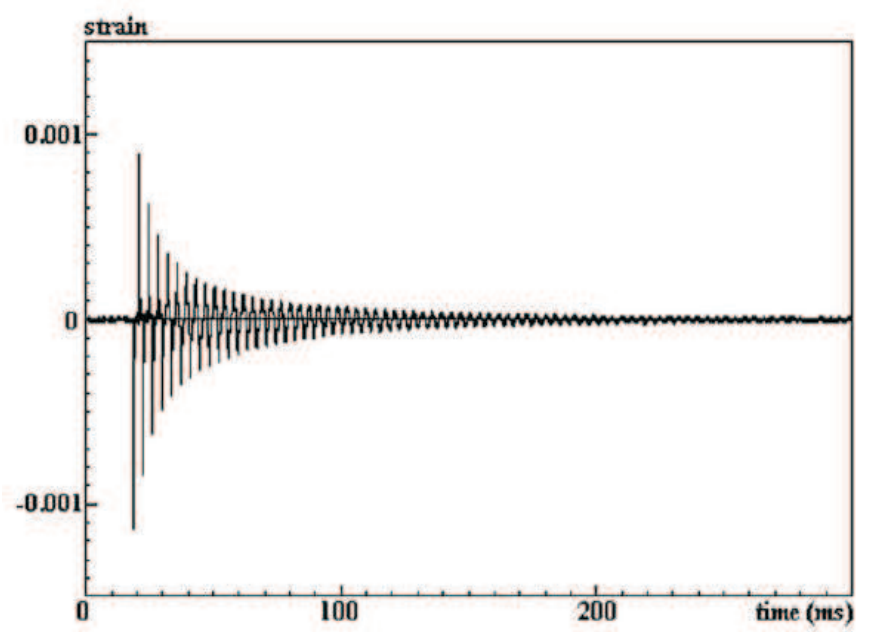

Fig. 32 Recorded strain in the Nylon bar specimen. Long-time record of strain pulses. 


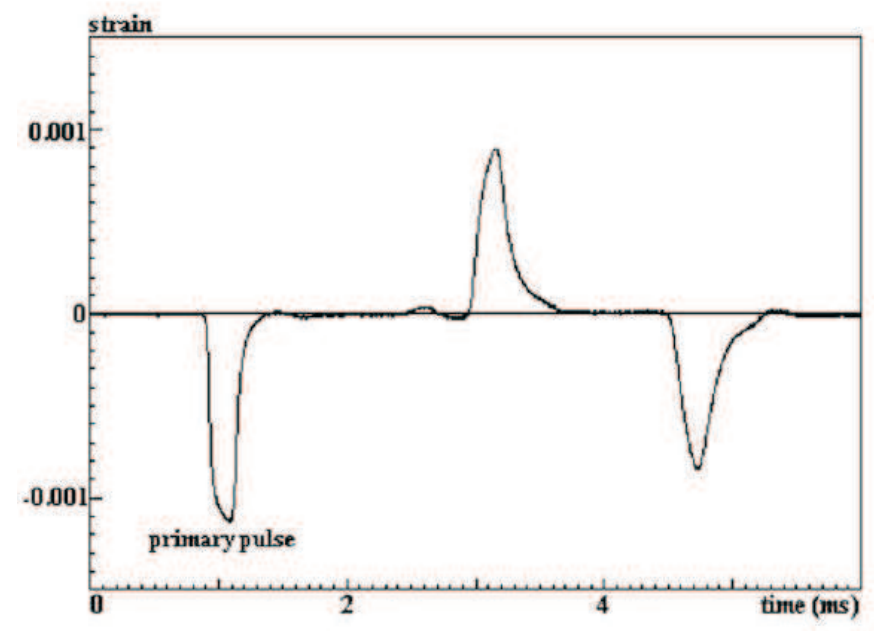

Fig. 33 Primary strain pulse followed by strain pulses which have undergone one and two free-end reflections

The spectrum $\left|\hat{\varepsilon}_{b}^{\infty}\right|$, computed from the long-time strain record, is shown in Fig. 34, 35 and 36. It is easily checked that resonance peaks are clearly identified. In figures 35 and 36 , the experimental peak is shown with the theoretical one identified by the minimization process described above (in figure 35 , the superposition is perfect at the scale of the drawing).

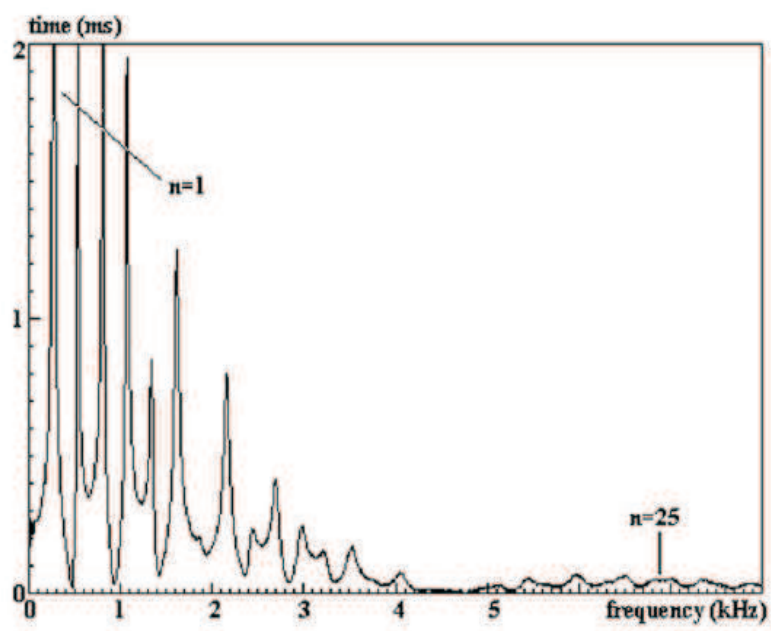

Fig. 34 Experimental spectrum 


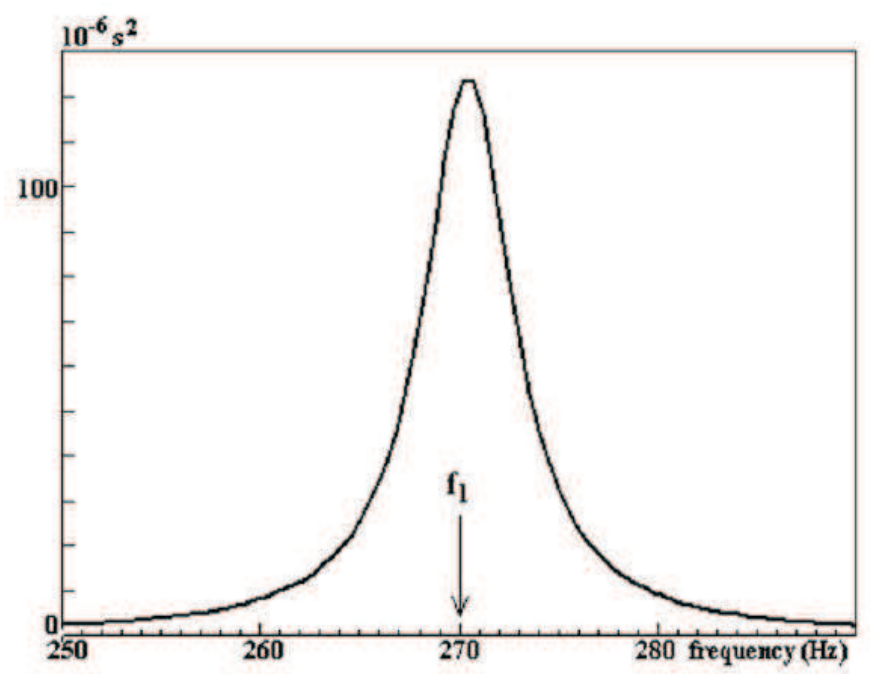

Fig. 35 Spectrum, zoom on $\mathrm{f}=270 \mathrm{~Hz}$

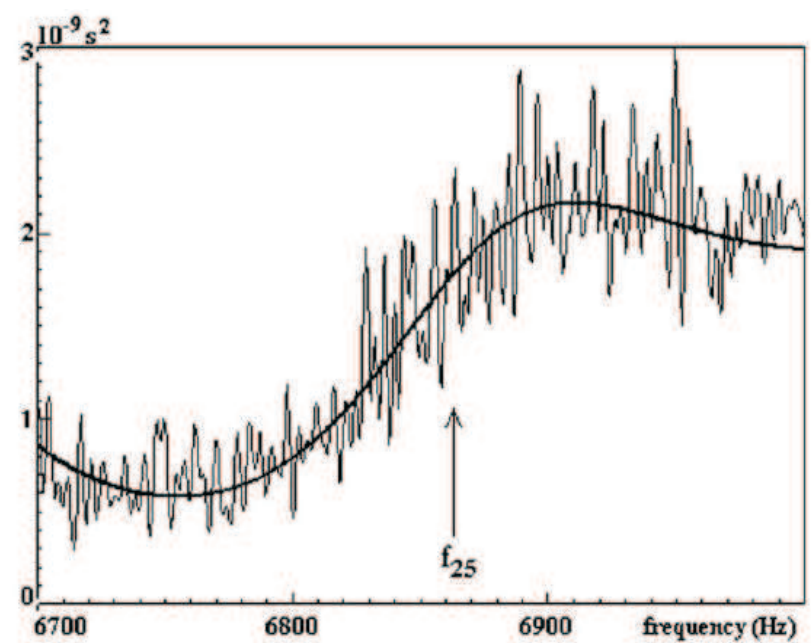

Fig. 36 Spectrum, zoom on $\mathrm{f}=6860 \mathrm{~Hz}$

Corresponding to the peaks, a set of experimental points is obtained which provides, for each frequency, a value for wave speed and damping (circles in figures 37,38 ) 


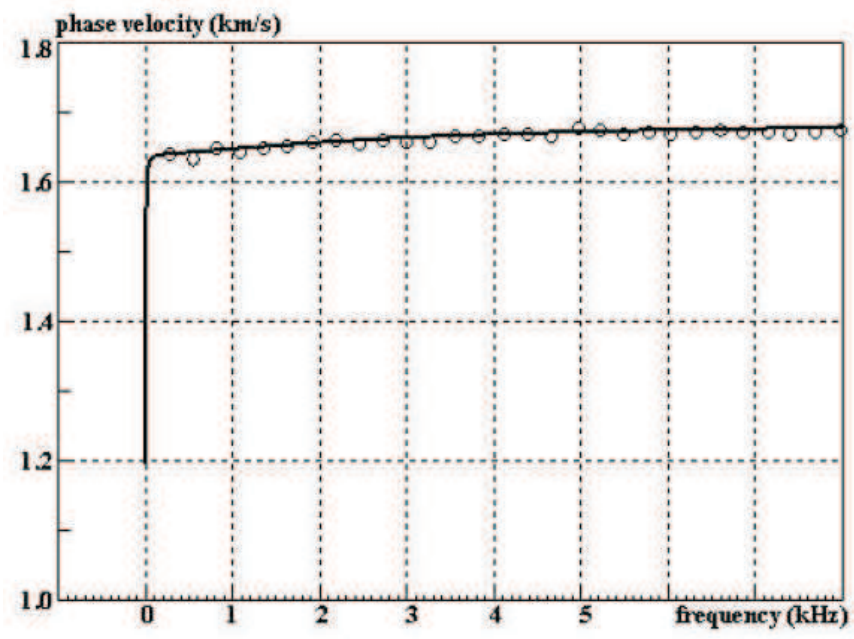

Fig. 37 Wave speed

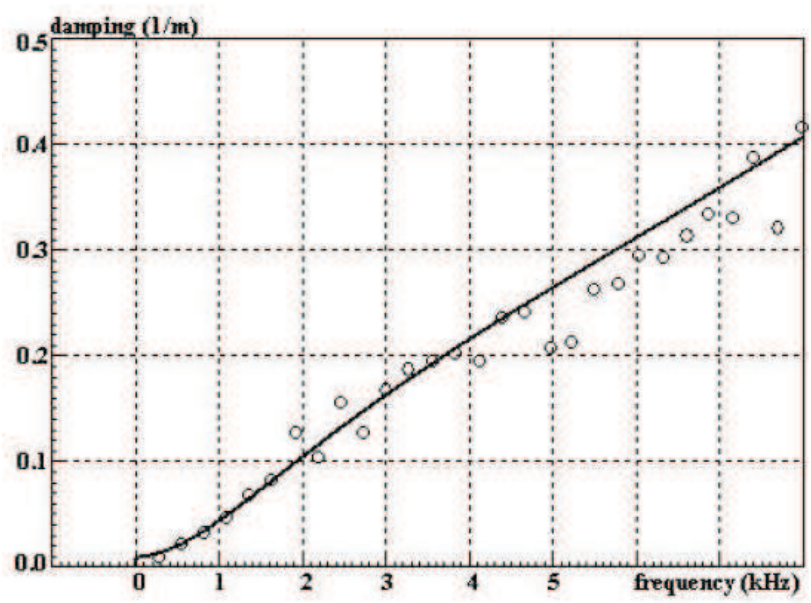

Fig. 38 Damping

By averaging, the results in figures 37 and 38 immediately provide the dispersion relations that could be used right away for the wave shifting.

Using the mathematical analysis presented by Collet et al. (2012), an optimal rheological model type is built and identified with 7 springs and dashpots. Note that the number of elements is not an assumption - as was done in Othman et al. (2001) - but is a result of the method. Knowing the 
corresponding Young's modulus, wave speed and damping as functions of frequency are then built. In the present example, they correspond to black continuous curves in figures 37 and 38 .

\section{Long-time measurements}

Basic measuring techniques involving the use of bars require the knowledge of the two elementary waves which propagate in opposite directions. Once they have been characterised, they can be shifted to the appropriate crosssections (the bar specimen interfaces for example) and all the mechanical values required can be calculated. The SHPB technique involves the independent measurement of each wave.

Some authors - Lundberg \& Henchoz (1977), Yanagihara (1978), Zhao \& Gary (1997), Jacquelin \& Hamelin (2001) - have carried out two measurements on each bar. The pioneers in this respect were Lundberg \& Henchoz (1977) and Yanagihara (1978), who independently developed a wave separation technique based on one-dimensional wave propagation theory. Their methods didn't take into account the wave dispersion. Some attempts have been made to develop methods also accounting for the wave dispersion, starting with Zhao \& Gary (1997). However, the solutions proposed so far are sensitive to noise. A wave separation technique based on multiple measurements has been proposed by Othman \& al. (2001) and Bussac \& al. (2002). In line with Jacquelin and Hamelin (2003), this method will be subsequently called the BCGO method. It is based on the Maximum Likelihood approach and involves performing multiple strain measurements on each bar. This method, which is not noise-sensitive, requires an extra velocity measurement at very low strain rates. Jacquelin \& Hamelin (2003) have developed an alternative three-point wave separation technique which is also insensitive to noise but the gauges are cemented to specific points and the force is calculated at one bar end (which is the only point where it can be calculated). The BCGO method is illustrated with the analysis of Split Hopkinson Bar tests, and an extended range of strain-rates (from $10^{-1}$ to $510^{3} \mathrm{~s}-1$ ) for the study the rate sensitivity of an aluminum alloy.

\subsection{Wave separation: the BCGO method}

In this section, the BCGO method is briefly presented.

Let us consider an elastic or viscoelastic bar with length $l$. In the case of single mode propagating longitudinal waves, the stress, strain, displacement and velocity are expressed in terms of the Fourier transforms as follows: 


$$
\begin{array}{r}
\tilde{\varepsilon}(x, \omega)=A(\omega) e^{-i \xi(\omega) x}+B(\omega) e^{i \xi(\omega) x} \\
\tilde{\sigma}(x, \omega)=E^{*}(\omega)\left(A(\omega) e^{-i \xi(\omega) x}+B(\omega) e^{i \xi(\omega) x}\right) \\
\tilde{v}(x, \omega)=-\omega\left(A(\omega) e^{-i \xi(\omega) x}-B(\omega) e^{i \xi(\omega) x}\right) / \xi(\omega) \\
\tilde{u}(x, \omega)=i\left(A(\omega) e^{-i \xi(\omega) x}-B(\omega) e^{i \xi(\omega) x}\right) / \xi(\omega)
\end{array}
$$

where $A(\omega)$ and $B(\omega)$ are the Fourier components of the forward and downward waves, respectively, at the origin of the bar, $E *(\omega)$ is the complex Young's modulus and $\xi(\omega)=k(\omega)+i \alpha(\omega)$ is the complex wave number. Eqs. (23) show that the strain, stress, displacement and particle velocity can be obtained at any point on the bar if the following four parameters are known: $\xi(\omega), E^{*}(\omega), A(\omega)$ and $B(\omega)$.

The two parameters $E *(\omega)$ and $\xi(\omega)$ depend only on the bar characteristics (its geometry and material). They only need to be determined once. Here we used the method presented in chapter 7 .

In what follows, $E *(\omega)$ and $\xi(\omega)$ are therefore assumed to be known. $A(\omega)$ and $B(\omega)$ are calculated based on the data obtained by performing three strain measurements and one velocity measurement. We express the fact that the signals recorded are noisy by writing that they are the sum of the exact value of the strain (or the velocity) and an unknown noise. The statistical distribution of the noise is assumed to be Gaussian. Consequently, the Maximum Likelihood Method can be used to estimate the two functions $A(\omega)$ and $B(\omega)$. This consists in writing that the signals measured correspond to the most probable event. Our problem is therefore equivalent to the minimization of a functional: this minimization yields an explicit formula for $A(\omega)$ and $B(\omega)$ as a function of the material and geometric parameters and the measured quantities - see Bussac \& al. (2002).

The elementary functions $A(\omega)$ and $B(\omega)$ are then computed for each test. Using eqs. (23), one can now determine the force and the velocity at each point on the bar, especially at the bar ends. By applying the BCGO method to each of the bars, it is then possible to assess the force and the velocity at the two bar/specimen interfaces.

\subsection{Experimental set-up}

As an example, it is proposed to explore the strain-rate sensitivity of aluminium. The behaviour of this material is explored under a large range of strain rate conditions: quasi-static, medium and high strain rates.

In the high strain-rate tests, the classical time domain approach cor- 
responding to the Split Hopkinson Bar was used. In the quasi-static and medium strain-rate tests, the new method involving the use of extra sensors on the SHPB was adopted, as explained above. The corresponding apparatus is a modified SHPB system loaded by an actuator, of the kind first introduced by Zhao \& Gary (1997). In the quasi-static and medium strainrate tests, low velocity loading was required. The kinetic energy of a striker would not suffice to induce large strains in the specimen, and longer loading durations were required. The bar system was therefore loaded using a hydraulic actuator and selecting the speed as required. Low loading speeds (of less than $0.1 \mathrm{~m} / \mathrm{s}$ ) can be monitored and automatically kept constant throughout the test. At higher speeds of up to $5 \mathrm{~m} / \mathrm{s}$, the requisite value is maintained approximately constant during the test. The new apparatus is called the "slow bars" apparatus. In the present case, three strain gauges and an optical displacement extensometer were used on each bar. The derivative of the displacement was then calculated numerically to obtain the velocity. A simplified scheme is presented in Fig. 39 and pictures of the set-up are given in figures $40 \mathrm{a}$ and $40 \mathrm{~b}$.

Aluminium bars $40 \mathrm{~mm}$ in diameter and $3 \mathrm{~m}$ in length were used in these tests on the strain-sensitivity of the aluminium.

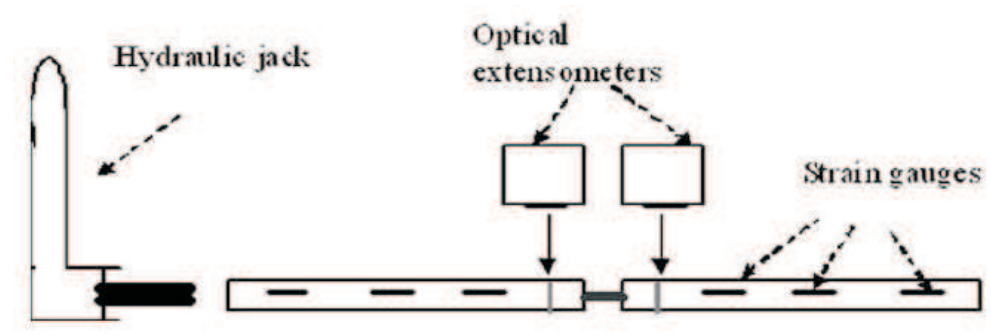

Fig. 39 Simplified scheme of the "slow bar" set-up 

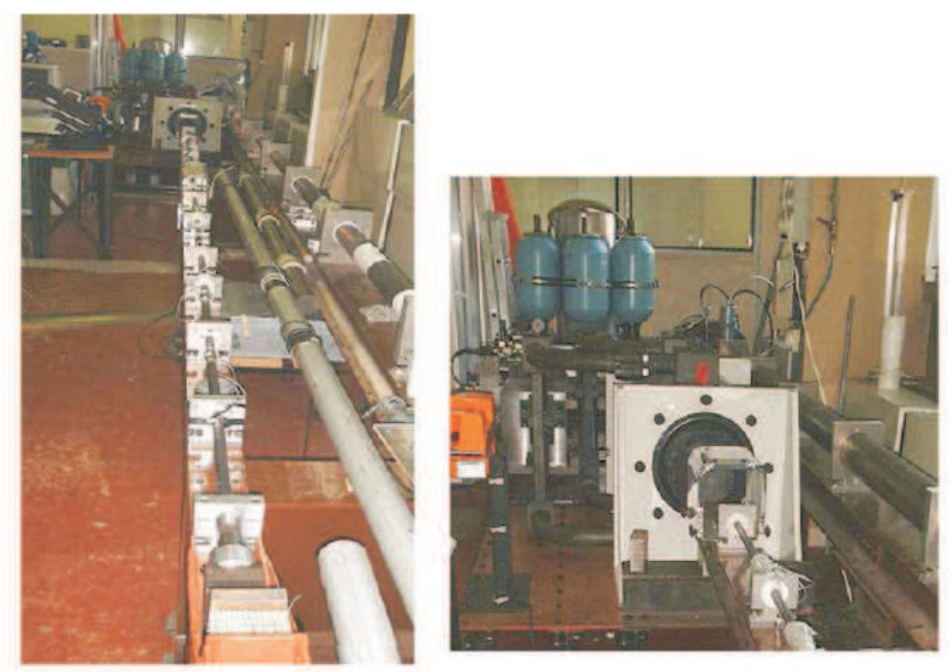

Fig. 40a View of the slow bar rig Fig. 40b Hydraulic actuator

Both the Hopkinson bar and the slow bars give force and displacement measurements at the two bar/specimen interfaces. To determine the behaviour of the material, we assume the stress and strain to be homogeneous in the specimens. This assumption was systematically checked in the case of the slow bars and SHPB by making sure that the forces measured in the bars on each side of the specimen were practically equal. As was to be expected, it was observed that the smaller the loading rate, the more exactly this condition (called equilibrium) was fulfilled.

\subsection{A bar-bar test check}

To check the consistency of the complete system (as well as testing the accuracy of the BCGO method), a bar/bar test was carried out, in which the two bars were put in contact without placing a specimen between them. The force and the displacement were computed at the bar/bar interface in two independent ways, using the measurements obtained on each bar separately and checking whether the two results obtained were equal. In the present example, the velocity of the hydraulic jack was set at $1.5 \mathrm{~m} / \mathrm{s}$.

The forces and displacements calculated at bar ends are compared in Fig. 41 and Fig. 42, respectively. The results of computations made on each bar were almost equal, as was to be expected (with the bars in contact). 


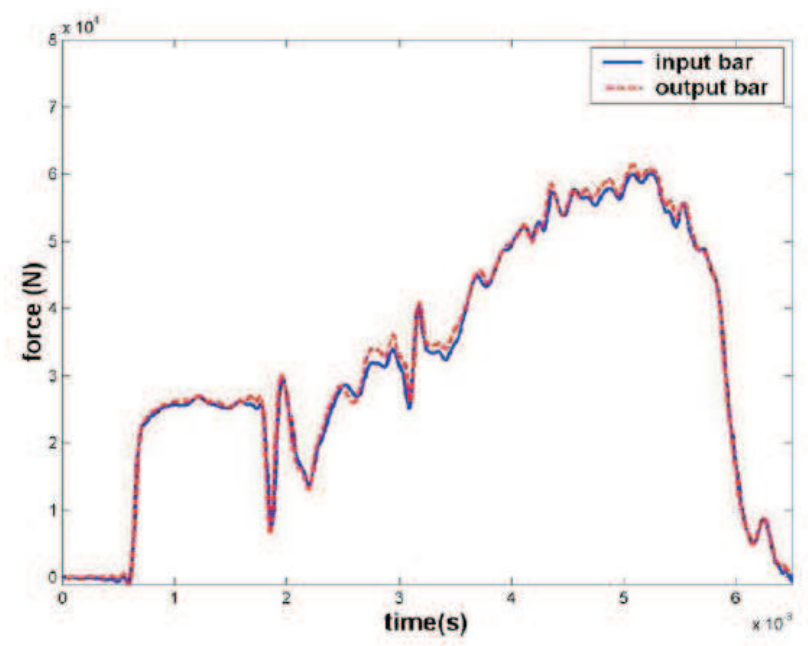

Fig. 41 Forces at the bar/bar interface.

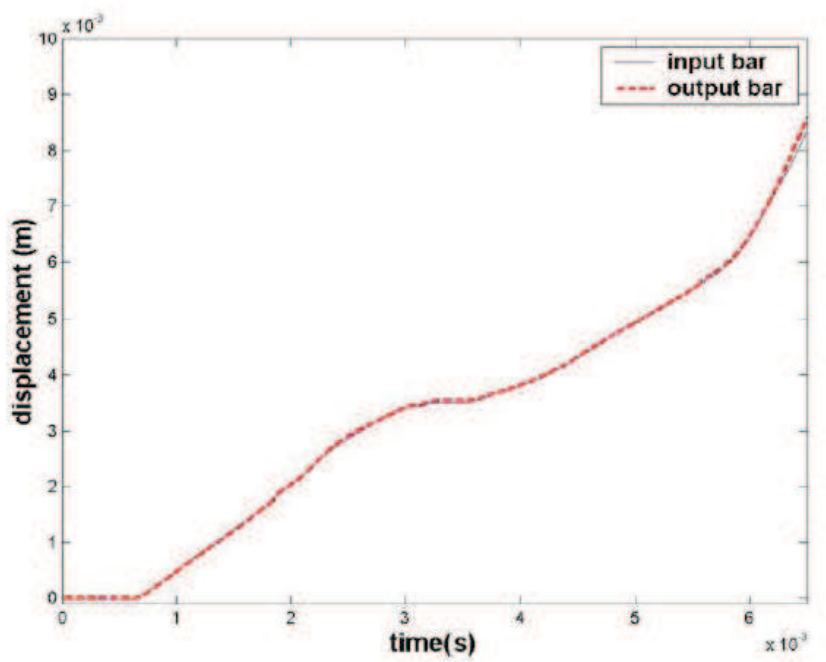

Fig. 42 Displacements at the bar/bar interface.

\subsection{Aluminium characterisation}

In the slow bar tests, the specimens used were $6 \mathrm{~mm}$ in length and $6 \mathrm{~mm}$ in diameter. The velocity of the hydraulic jack ranged from $2.10^{-4}$ to $2.5 \mathrm{~m} / \mathrm{s}$. The strain rate ranged approximately from $10^{-1}$ on to $400 / \mathrm{s}$. In Hopkinson bar tests, the specimen geometry and the striker impact velocity have to 
be adapted to the strain rates, which ranges approximately from 150 to $5000 / \mathrm{s}$. In each test, the assumption that the force equilibrium conditions were satisfied between the two bar/specimen interfaces was checked. An example is given in Fig. 43 in the case of slow bars. The forces dropped suddenly at approximately $0.032 \mathrm{~s}$ because the specimen broke at that point.

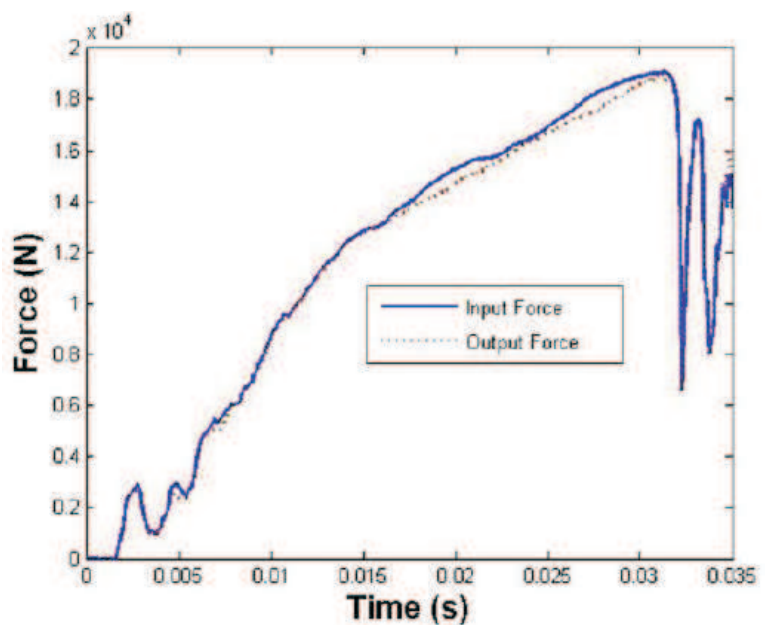

Fig. 43 Specimen equilibrium.

Displacement data were also calculated at each of the specimen faces, as shown in Fig. 44.

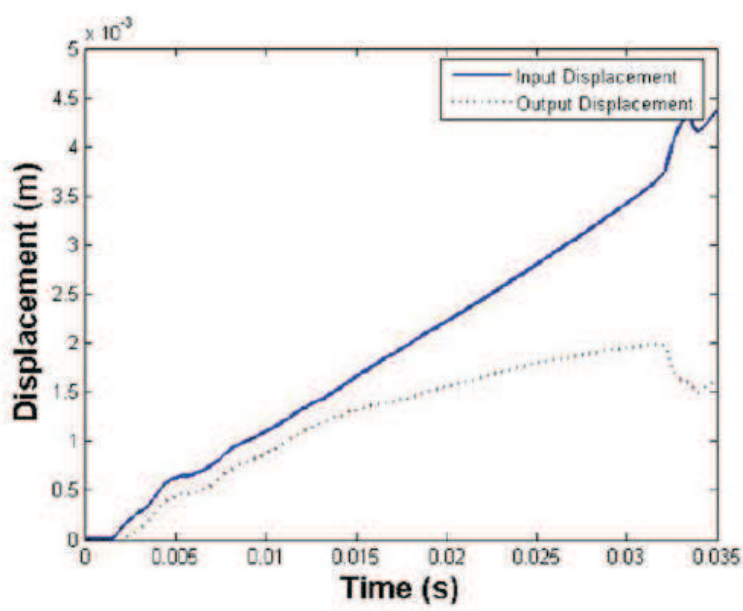


Fig. 44 Displacements at specimen faces.

Assuming (as well as checking) that the equilibrium conditions were satisfied, the stresses, strains and strain rates were obtained from these measurements. It can be noted that the duration of the tests increased considerably when using slow bars, reaching several seconds, in comparison with the usual duration of 500s in the case of the classical Hopkinson bar tests carried out on the same bars.

The results of the aluminum test show that this material is only slightly sensitive to the strain rate. In Fig. 45, the changes in the stress corresponding to a $10 \%$ strain level were plotted versus the strain rate corresponding to the same strain level. The stress increased by approximately $15 \%$ when the strain rate increased from $10^{-1}$ to $5000 / \mathrm{s}$. Some slow bar tests and Hopkinson bar tests were also both carried out at strain rates in the 150 to $400 / \mathrm{s}$ range. The mean difference between the results obtained with these two methods was less than 20MPa.

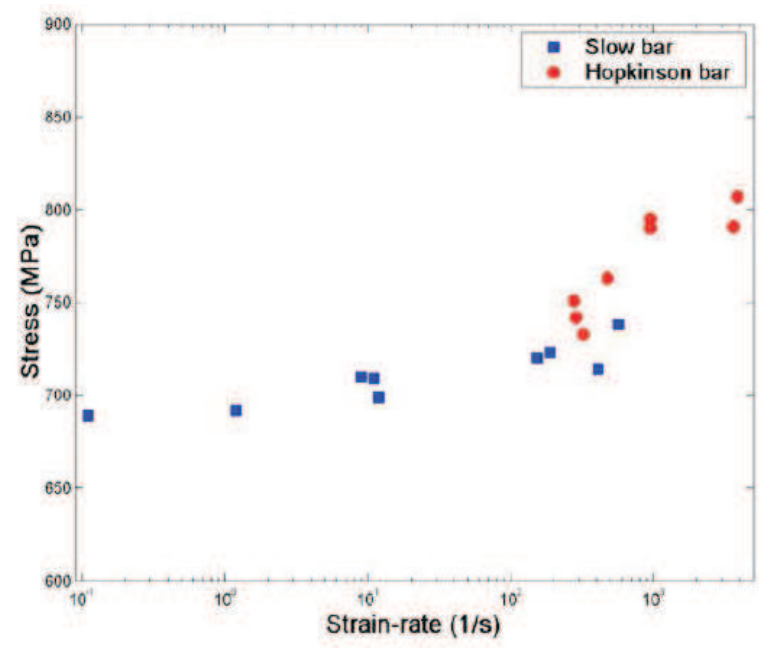

Fig. 45 Strain-rate sensitivity of aluminum.

\subsection{A powerful tool}

An easy interrupted test.

A simple way to make an interrupted test is to ensure that, after the first loading, the speed of the output bar at specimen side is larger than the speed of the intput bar at specimen side. This test also needs, of course, that the output bar is free of moving, that provides an extra "strain" measurement 
at its end (null strain). The total strain amplitude is then limited by the striker length (provided that the specimen behaviour is estimated as it also governs the strain rate). This will happen, when the transmitted wave will be greater, in amplitude, than half of the incident strain (with bars of the same impedance).

A test have been designed for this purpose, with aluminium bars (diameter $40 \mathrm{~mm}$, in input bar $1.8 \mathrm{~m}$, output bar $1.3 \mathrm{~m}$, striker $0.8 \mathrm{~m}$ - speed 10 $\mathrm{m} / \mathrm{s})$.

The corresponding wave records are shown in fig. 46 and 47 . One extra gauge has been added on each bar and the record duration has been increased in order to check the validity of the "interrupted test" assumption.

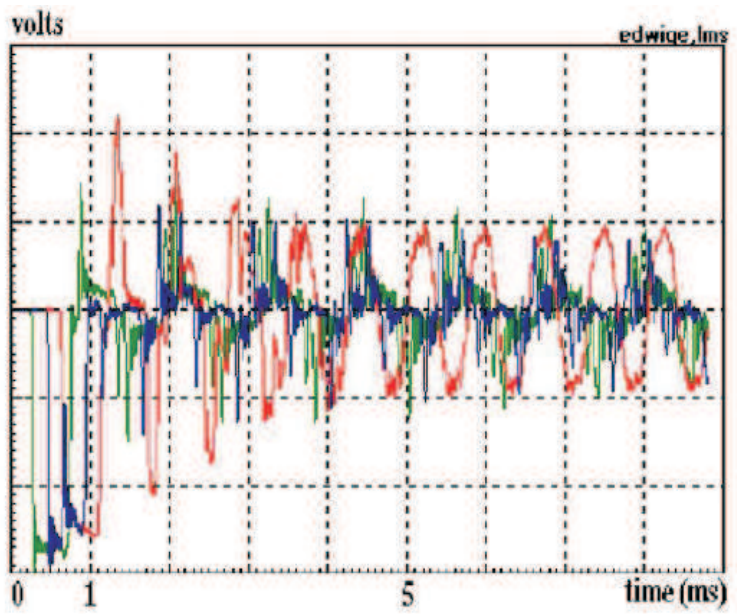

Fig. 46 Recorded waves. 


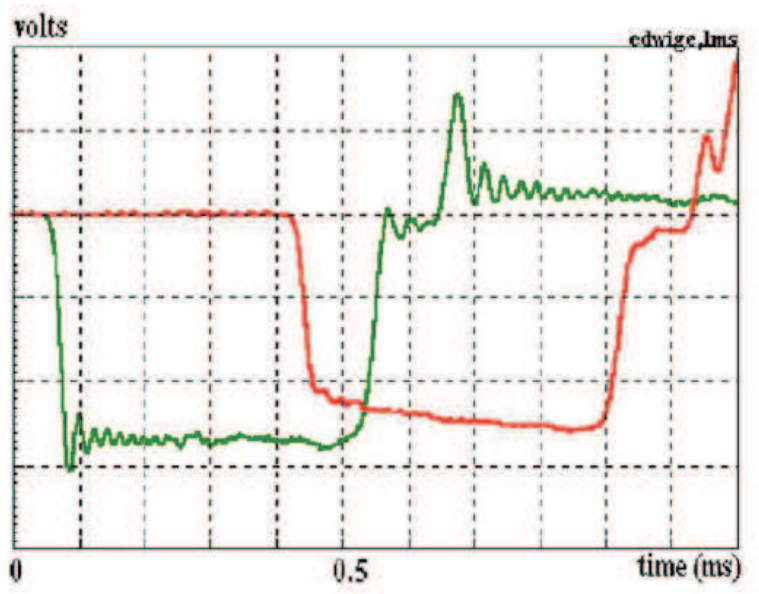

Fig. 47 Recorded waves (early instants).

Using the BCGO method, displacements computed at bar ends (specimen side) prove that the test was indeed an "interrupted test" as the specimen could not be reloaded, as shown in figs. 48 and 49 .

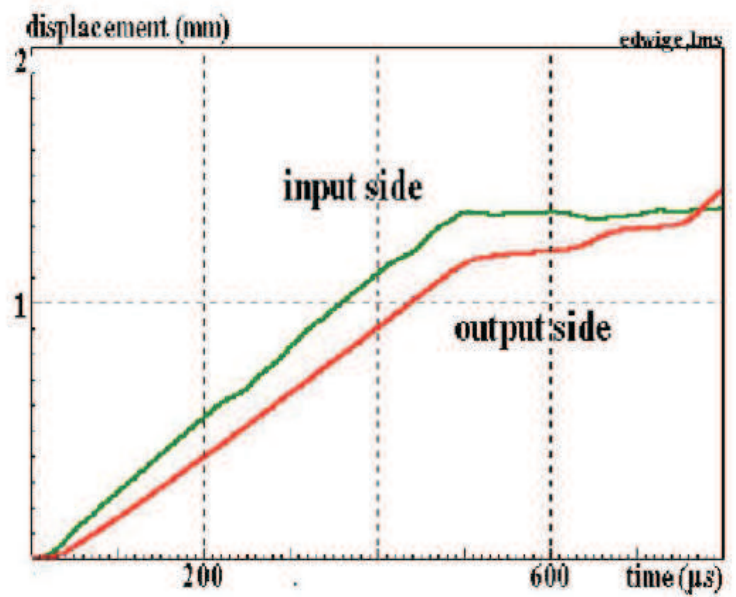

Fig. 48 Displacements at bar ends (specimen side) - short time 


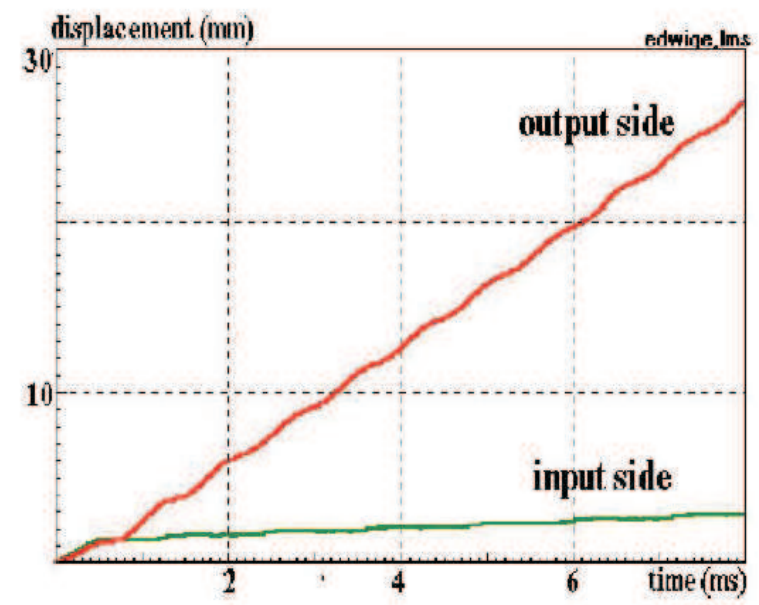

Fig. 49 Displacements at bar ends (specimen side) - long time

The BCGO method is valid at quasi-static strain-rates.

As the method works in the frequency domain and as the size of numerical records is limited, the data acquisition frequency used at very low strain rates is no more than $1 \mathrm{kHz}$. Considering that the corresponding time base corresponds to metricconverterProductID $5 \mathrm{~m} 5 \mathrm{~m}$ of an elastic wave propagation, one could fear that the description of waves in bars is insufficient.

This is not the case, as demonstrated in fig. 54 where a test at a loading speed of $5 \mathrm{~mm} / \mathrm{s}$ is shown. The specimen is a water cylinder contained in a pressure vessel in which the bars act as pistons. 


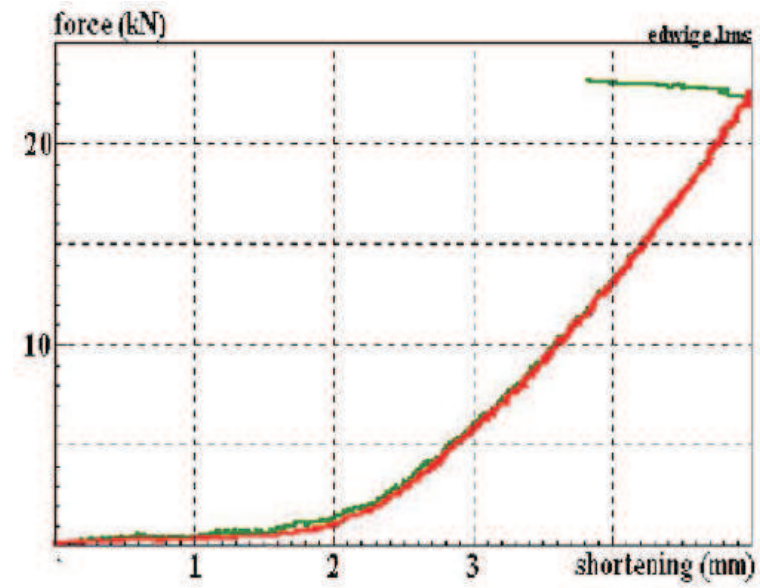

Fig. 50 Comparison of the BCGO method with a quasi-static analysis

For the quasi-static analysis, the displacement of each bar is corrected with the mean strain.

$$
\varepsilon_{m}=\left(\varepsilon_{1}+\varepsilon_{2}+\varepsilon_{3}\right) / 3 \quad d_{e}=d_{\text {meas }}+\varepsilon_{m} d\left(x_{\text {meas }}, \text { specimen }\right)
$$

The force is simply deduced from the mean strain.

$$
F=S_{b} E_{b} \varepsilon_{m}
$$

\section{Conclusion}

Most problems related to dynamic testing have been addressed here, in particular those dealing with bars that are very commonly used at high strain rates, from $200 / \mathrm{s}$ to $5000 / \mathrm{s}$.

Higher strain-rates have not been studied as they generally involve very expensive equipments.

On the other strain-rate side, some suggestions have been proposed to extend the use of bars at medium strain-rates (1/ to $200 / \mathrm{s}$ ) where no industrial experimental method is easily available.

Recall that dynamic material testing needs to keep in mind a few points:

- Direct measurements of specimen mechanical properties like strains and stresses are almost impossible.

- Forces and displacements must be done (or computed) as close as possible to specimen faces. 
- Going from global measurements to mechanical properties needs to evaluate dynamic effects (stresses and strains homogeneity, lateral inertia, temperature increase) in order to quantify the subsequent approximation.

Some modern optical methods which allow for direct measurements during high rates of testing, like image correlation for a more local estimation of the strain and infra-red temperature measurements, are welcome when available, in order to confirm and complete the validity of this difficult process that leads, from global forces and displacements measurements, to some knowledge of the material behaviour.

\section{Bibliography}

Achenbach, J.D.(1978), Wave propagation in elastic solids., North-Holland publishing company.

Bacon, C., An experimental method for considering dispersion and attenuation in a viscoelastic Hopkinson bar, Experimental Mechanics, 36, n 4 (1998) 242-249.

Bancroft, D., (1941) The velocity of longitudinal waves in cylindrical bars. Phys. Rev. 59, 588-593.

Blanc, R. H., (1971). Dtermination de l'quation de comportement des corps visco-lastiques linaires par une mthode d'impulsion. Ph. D. Thesis, Universit d'Aix-Marseille, published in part in Problmes de PersonNameProductIDla Rh?ologiela Rh?ologiela Rhologie (W.K. Nowacki, editor), 65-85. IPPT PAN, CityplaceWarsaw

Bussac M.N.., Collet P., Gary G., Othman R., "An optimisation method for separating and rebuilding one-dimensional dispersive waves from multipoint measurements. Application to elastic or viscoelastic bars," J. Mech. Phys. Solids, 50, 321-350 ( 2002).

Bussac, M.,N.,Collet, P., CityplaceGary, G., Lundberg, B. Mousavi, S., (2008) Viscoelastic impact between a cylindrical striker and a long cylindrical bar Int J. of Impact Engineering. Vol 35 (5), 2008, 226-239

Collet P., Lundberg B., Gary G., Mohr D., (2012) Complex Modulus Estimation Respecting Causality: Application to Viscoelastic Bars. DYMAT, placeFreiburg, accepted for publication

Collet P., Lundberg B., Gary G., (2012) Noise-corrected Estimation of Complex Modulus in Accord With Causality and Thermodynamics: Application to an Impact test. J. of App. Mechanics, accepted for publication Cristescu, N. (1967). Dynamic Plasticity. North-Holland, CityplaceAmsterdam.

Davies, R.M. (1948), A critical study of Hopkinson pressure bar. Phil. 
Trans. Roy. Soc., A240, 375-457.

Davies E.D.H., Hunter S.C., (1963), The dynamic compression testing of solids by the method of the split Hopkinson pressure bar. J. Mech. Phys. Solids, 11, 155-179.

Chree, C. (1889) The equations of an isotropic elastic solid in polar and cylindrical coordinates, their solutions and applications. CityplaceCambridge Phil. Soc., Trans. 14, 250-369.

Follansbee, P.S. and Franz, C. (1983) Wave propagation in the split Hopkinson pressure bar. J. Engng. Mater. Tech., 105, 61-66.

Forquin,P., Safa, K., Gary, G. (2010) Influence of free water on the quasistatic and dynamic strength of concrete in confined compression tests, Cement and Concrete Research, 40, 2, February 2010, 321-333

Gary, G., Klepaczko, J., R., Zhao, H. (1991) Correction de dispersion pour l'analyse des petites dformations aux barres de Hopkinson, Journal de Physique IV, 1(1991), C.3, 403-410.

Gary, G., Nowacki, W.,K.(1994). Essai de cisaillement plan appliqu des tles minces, Journal de Physique IV, 4(1994), C8, 65-70

Gary.,G. and Bailly.,P., (1998), Behaviour of quasi-brittle material at high strain rate. Experiment and modelling, European Journal of Mechanics A/Solids, 17, 3, (1998), 403-420.

CityGary, G. (2001), Some aspects of dynamic testing with wave-guides, New Experimental Methods in Material Dynamics and Impact, Trends in Mechanics of Materials, Volume 3, W.K.Nowacki and J.R.Klepaczko (Eds), INB ZTUREK, addressStreetP.O.Box 374, 00-950 CityWarsaw 1, countryregionplacePoland, 2001, 179-222

Gong, J.C. Malvern, L.E. and Jenkins, D.A. (1990) Dispersion investigation in the split Hopkinson pressure bar. J. Engng. Mater. Tech., 112, 309-314. Gorham, D.A. (1983) A numerical method for the correction of dispersion in pressure bar signals. J. Phys. E: Sci. Instrum. 16, 477-479

Hillstrm, L., Mossberg,M; and Lundberg, B. (2000) Identification of complex modulus from measured strains on an axially impacted bar using least squares. Journal of Sound and Vibration, 230(4), 689-707.

Jacquelin, E. and Hamelin P., (2001) "Block-bar device for energy absorption analysis," Mech. Systems Signal Processing, 15, 603-617 (2001).

Jacquelin, E. and Hamelin, P., "Force recovered from three recorded strains," Int. J. Solids Structures, 40, 73-88 (2003).

Klepaczko, J., R., (2007) Introduction to experimental techniques for materials testing at high strain rates. Strength of materials under impact, PlaceTypeplaceInstitute of PlaceNameAviation ed. ,Al. Krakowska 110/114, 02-256 WARSAW, POLAND

Lifshitz J.M., Leber H., (1994), Data processing in the split Hopkinson 
pressure bar tests. Int. J. Impact Engng., 15, 723-733.

Lundberg, B. and Henchoz, A.,(1977) "Analysis of elastic waves from twopoint strain measurement," Exper. Mech., 17, 213-218 (1977).

Lundberg, B. and Blanc, R. H. (1988) Determination of mechanical material properties from the two-point response of an impacted linearly viscoelastic rod specimen, J. Sound and Vibration, 126, 97-108.

Malinowski, J.,.Z., Klepaczko, J.,R., (1986) A unified analytic and numerical approach to specimen behaviour in the Split-Hopkinson pressure bar, International Journal of Mechanical Sciences, 28, 6, (1986), 381-391

Malvern L.E., (1951), Propagation of longitudinal waves of plastic deformation. J. Appl. Mech., 18, 203-208.

Merle. R., \& Zhao., H. (2006), On the errors associated with the use of large diameter SHPB, correction for radially non-uniform distribution of stress and particle velocity in SHPB testing, International Journal of Impact Engineering 32 (2006) 1964-1980.

Mohr, D., Gary, G.; Lundberg, B.; (2010) Evaluation of Stress-strain Curve Estimates in Dynamic Experiments, IE-D-08-00215R1, International Journal of Impact Engineering 37, 2010, 161-169

Negreanu, I., Gary, G., Mohr, D., Enhanced infrared radiation method for temperature measurement in dynamic experiments, DYMAT 2009, EDP Sciences, 2009, 179-183

Othman R., Blanc R.H., Bussac M.N., Collet P., Gary G., (2001), A spectral method for wave dispersion analysis. Application to an aluminium rod. Proc. 4th Int. Symp. on Impact Engng, CityplaceKumamoto, countryregionJapan.

Othman R., Bussac M.N., Collet P., Gary G., "Sparation et reconstruction des ondes dans les barres lastiques et viscolastiques partir de mesures Redondantes, " Comptes Rendus de l'Acadmie des Sciences, Srie IIb, 329, 369-376 (2001).

Pochhammer L., (1876), Uber die fortpflanzungsgeschwindigkeiten kleiner schwingungen in einem unbergrenzten isotropen kreiszylinder. J. fr die Reine und Angewandte Mathematik, 81, 324-336.

Rittel, D., (1999) On the conversion of plastic work to heat during high strain rate deformation of glassy polymers, Mechanics of Materials, 31, 2, February 1999, 131-139.

placeRota, L., (1994) An inverse approach of identification of dynamic constitutive equations, International symposium on inverse problems, Ed. Balkema

Safa, K.; Gary, G.(2010); Displacement correction for punching at a dynamically loaded bar end, IE-1835, International Journal of Impact Engineering $37,2010,371-384$ 
Safford. N., A., (1992) Laterial testing up to $10^{5} \mathrm{~s}^{-1}$ using a miniaturized Hopkinson bar with dispersion corrections, Proc. $2^{\text {nd }}$ Intl. Symp. On Intense Dynamic Loading and its Effects, PlaceNameSichuan PlaceTypeUniversity Press, CityplaceChengdu, country-regionChina, (1992), 378

Sokolovsky V.V, (1948), The propagation of elastic-viscous-plastic wave in bars. Prikl. Mat. Mekh., 12, 261280.

Tyas A., Watson A. (2001) An investigation of frequency domain dispersion of pressure signals. Int. J. Impact Engng., 25, 87-101

Yanagihara, N., (1978) "New measuring method of impact force," Bull. Jpn. Soc. Mech. Engng., 21, 1085-1088 (1978).

Yew E.H. and Chen C.S., (1978) Experimental study of dispersive waves in beam and rod using FFT, J Appl. Mech., 45, 940-942.

Zhao, H., and Gary, G., (1995) A three dimensional longitudinal wave propagation in an infinite linear viscoelastic cylindrical bar. Application to experimental techniques, J. Mech. Phys. Solids, 43(1995), 1335-1348

Zhao, H., Gary, G., (1996) On the use of SHPB technique to determine the dynamic behavior of the materials in the range of small strains, Int. J. Solid. \& Structure. 33(1996), 3363-3375.

Zhao, H and Gary, G., (1997) "A new method for the separation of waves. Application to the SHPB technique for an unlimited measuring duration," J.Mech.Phys.Solids, 45, 1185-1202 (1997). 